\title{
Theta-band functional connectivity in the dorsal fronto-parietal network predicts goal-directed attention
}

\author{
Julia Fellrath ${ }^{1,2}$, Anaïs Mottaz ${ }^{1}$, Armin Schnider $^{1,2}$, Adrian G. Guggisberg $^{1,2}$ and \\ Radek Ptak ${ }^{1,2,3}$
}

${ }^{1}$ Laboratory of Cognitive Neurorehabilitation, Faculty of Medicine, University of Geneva, Switzerland

${ }^{2}$ Division of Neurorehabilitation, Department of Clinical Neurosciences, University Hospitals Geneva, Geneva, Switzerland

${ }^{3}$ Faculty of Psychology and Educational Sciences, University of Geneva, Geneva, Switzerland

\section{Corresponding author:}

Julia Fellrath, PhD

Division of Neurorehabilitation, Geneva University Hospitals and Faculty of Medicine, University of Geneva

26, Av. de Beau-Séjour, 1211 Geneva 14, Switzerland

Julia.Fellrath@hcuge.ch 


\section{ABSTRACT}

Functional imaging studies have identified a dorsal fronto-parietal network whose activity reflects shifts of attention in space and is sensitive to the behavioural relevance of stimuli. In patients with severe deficits of spatial attention this network is often structurally preserved. Here, we show that resting-state EEG functional connectivity in the dorsal fronto-parietal network predicts impaired goal-directed processing in stroke patients with spatial attention deficits. Eleven right-hemisphere damaged patients with different degrees of contralesional spatial deficits and sixteen age-matched healthy controls performed a visuo-spatial task which required them to react to a central target while ignoring task-relevant distracters presented left or right of fixation. Unlike controls, performance of patients was not modulated by the goalrelevance of peripheral distracters. Compared to controls patients showed a significant decrease in theta-band connectivity between the right dorsolateral prefrontal cortex and the right superior parietal region. Moreover, in both groups we observed a significant correlation between fronto-parietal connectivity and the behavioural effect of distracter relevance. These findings indicate that fronto-parietal functional connectivity is impaired in patients with spatial attention deficits and predicts effects of goal-relevant information on target processing.

Keywords: Spatial attention; Goal relevance; Functional connectivity; EEG; Fronto-parietal network; Theta-band. 


\section{INTRODUCTION}

Neuroimaging and human lesion studies have identified right-hemispheric fronto-parietal networks that are crucially involved in spatial attention. Unilateral spatial neglect is a disabling neurological disorder that is frequently associated with right hemispheric brain damage (Gainotti, Messerli, \& Tissot, 1972; Oxbury, Campbell, \& Oxbury, 1974; Weintraub \& Mesulam, 1987). It is characterized by the inability to detect, attend or respond to stimuli in spatial locations contralateral to the side of cerebral damage (Halligan, Fink, Marshall, \& Vallar, 2003; Heilman, Watson, \& Valenstein, 1993). Many neglect symptoms are related to the notion of impaired attentional orienting. Based on behavioural and neuroimaging studies, Corbetta and Shulman (2002) proposed a model in which they hypothesized that attentional operations during sensory orienting are carried out by two separate fronto-parietal systems: a dorsal attention system and a ventral attention system. According to the model, the dorsal system is involved in directing top-down attention based on goals and expectations and relies on parts of the intraparietal sulcus (IPS) and the dorsolateral premotor cortex, including the frontal eye field (FEF). The ventral system comprises the right temporal-parietal junction (TPJ) and the right ventral frontal cortex (VFC) and is sensitive to new and salient stimuli, especially when they appear at unexpected locations. A majority of lesion mapping studies found that lesions causing neglect frequently involve the right inferior parietal lobule (Committeri et al., 2007; Golay, Schnider, \& Ptak, 2008; Mort et al., 2003; Vallar \& Perani, 1986; Vossel et al., 2011), the temporo-parietal junction (Golay, et al., 2008; Grandjean, Sander, Lucas, Scherer, \& Vuilleumier, 2008; Karnath, Himmelbach, \& Kuker, 2003; Ticini, de Haan, Klose, Nagele, \& Karnath, 2010) or the superior temporal gyrus (Committeri, et al., 2007; Karnath, Berger, Kuker, \& Rorden, 2004; Karnath, Ferber, \& Himmelbach, 2001; Samuelsson, Jensen, Ekholm, Naver, \& Blomstrand, 1997). Thus, right brain lesions causing neglect do not seem to involve the dorsal attention network. This is in agreement with the observation that neglect patients appear to be more impaired in detecting salient stimuli than in top-down goal-directed orienting (Corbetta \& Shulman, 2002). For instance, they may direct their attention to the contralesional hemifield when asked to do so (Bartolomeo, Sieroff, Decaix, \& Chokron, 2001; Natale, Posteraro, Prior, \& Marzi, 2005). However, this appears to depend on the task, as other studies observed impaired reorienting of attention following presentation of behaviourally relevant stimuli, but not salient and irrelevant items (Ptak \& Golay, 2006; Ptak \& Schnider, 2006; Snow \& Mattingley, 2006). Similarly, visual search studies revealed impaired deployment of controlled attention towards the contralesional hemifield when patients searched for a combination of features (Aglioti, Smania, Barbieri, \& Corbetta, 1997; Esterman, McGlinchey-Berroth, \& Milberg, 2000; Fellrath, Blanche-Durbec, Schnider, Jacquemoud, \& Ptak, 2012; Riddoch \& Humphreys, 1987). Moreover, task-evoked fMRI responses in dorsal fronto-parietal cortex of patients with neglect exhibit abnormal 
interhemispheric coordination patterns, despite being structurally preserved (Corbetta, Kincade, Lewis, Snyder, \& Sapir, 2005).

Exploring goal-directed and stimulus-driven processes by examining functional connectivity patterns in healthy and brain-injured participants may provide insights for the understanding of spatial attention. Brain damage not only results in a loss of local neural function, but also affects remote networks, and these changes may remain undetected when analysing taskinduced activations or lesions of circumscribed brain areas (Alstott, Breakspear, Hagmann, Cammoun, \& Sporns, 2009; Honey \& Sporns, 2008). fMRI studies show that the connectivity between brain regions at rest has a correspondence with the topography of task-evoked brain activations (Mannan, Kennard, \& Husain, 2009; Zhao et al., 2011). However, functional connectivity at rest may provide additional information that is difficult to obtain from activation studies. In particular, functional connectivity statistically quantifies the interdependence between signals at different regions rather than treating each area independently (Varela, Lachaux, Rodriguez, \& Martinerie, 2001). This can provide more fine-graded insights into network interactions. Moreover, the strength of connectivity in a given network is linearly related to behavioural performance in tasks that depend on these networks (Baldassarre et al., 2014; Koyama et al., 2011; Wang et al., 2010). For example, He and colleagues (2007) used fMRI to investigate how disrupted functional connectivity in fronto-parietal attention networks may explain the spatial attention deficit characterizing neglect. The authors observed that functional connectivity recorded approximately one month after stroke was disrupted both in the ventral and the dorsal fronto-parietal attention network. Ventral network connectivity correlated with spatially non-lateralised attention deficits, while connectivity between left and right posterior parietal cortices correlated with the magnitude of the contralateral reorienting deficit. Thus, functional impairments of structurally intact neural networks may predict behavioural impairments in brain-injured patients.

The notion of functional connectivity may not only be applied to fMRI, but also to electroencephalography (EEG). Functional connectivity calculated between reconstructions of cortical oscillations obtained with inverse solutions allows measuring cortical network interactions (Niebur, Schuster, Kammen, \& Koch, 1991; Schoffelen \& Gross, 2009). In EEG studies, functionally relevant interactions can be obtained by assessing connections of each region of interest with the entire cortex. In addition, unlike fMRI EEG measures a rich spectrum of neural oscillations and may therefore capture additional information about functional brain organization. So far, only a few studies investigated the behavioural and clinical significance of functional connectivity impairments in brain-injured patients (Guggisberg et al., 2008; Martino et al., 2011; Nicolo et al., 2015). For example, a recent study found that the disruption of coherent electrical oscillations at rest predicts the 
magnitude of specific cognitive impairments, such as deficits of spatial working memory following stroke (Dubovik et al., 2012). These observations suggest that the magnitude of interregional EEG functional connectivity at rest is related to function and to behavioural performance irrespective of the precise locus of structural damage.

The present study aimed to investigate cortical network correlates of visual attention in healthy controls and right brain-damaged patients with varying degrees of spatial attention deficits. Reconstructed cortical networks based on surface EEG recordings allowed us to assess topographical and spectral changes in brain networks and to correlate them with measures of goal-directed and stimulus-driven attention.

\section{MATERIAL AND METHODS}

\subsection{Participants}

Eleven right-hemisphere damaged patients (2 females) and sixteen age-matched healthy controls ( 9 females) participated in the study. All participants gave written informed consent and the study was approved by the local ethical committee. Patients were examined while they were hospitalized following a first-ever stroke or haemorrhage. All participants were right-handed and had normal vision. All patients had preserved central visual fields as assessed with computerized perimetry or clinical confrontation testing. Table 1 shows demographic data and clinical characteristics of the patients. Both groups of participants had similar age $\left(\mathrm{t}_{25}=0.2\right)$. Given that we were interested in functional connectivity predictors of attention performance (as assessed with correlational measures) we included patients with varying degrees of spatial attention deficits. Inclusion criteria for patients were behavioural signs of left unawareness (difficulty with eating, dressing or in displacement) and leftlateralized failures in at least two of the following neglect tests: "Bells" cancellation (Gauthier, Dehaut, \& Joanette, 1989), reading of 40 words scattered pseudo-randomly on an A4-sheet of paper (Ptak, Di Pietro, \& Schnider, 2012), cancellation of inversed among upright "T"s (Ptak, Schnider, Golay, \& Muri, 2007) and line bisection (Ronchi, Posteraro, Fortis, Bricolo, \& Vallar, 2009). Time of behavioural testing (mean, 38 days post-injury), EEG recording (mean, 52.5 days post-injury) and of structural imaging (mean, 45.7 days post-injury) was comparable $\left(F_{2,30}=1.21\right)$.

Insert Table 1 about here 


\subsection{Go/no-go visuo-spatial task}

Visual attention was studied using a go/no-go task as described in a previous study (Fellrath, Manuel, \& Ptak, 2014). In short, subjects had to react to upright or rotated, central L- and Tshapes while ignoring lateral distracters briefly flashed in the left or right visual hemifield (Figure 1). The central stimulus was red, green or blue. For each individual subject one of these colours was randomly defined as the target colour throughout the experiment. Distracters were either relevant or irrelevant and had high or low saliency. Distracter saliency was defined as the difference in luminance between the distracter and the background (e.g., higher than background luminance for highly salient distracters). Distracter relevance was defined as the similarity between distracter and target regarding colour (e.g. "redness" when the target was red).

Figure 1B shows a schematic representation of the sequence of events in the task. Participants first fixated a small white cross $\left(0.5^{\circ}\right)$ shown in the middle of the screen. After $1000 \mathrm{~ms}$ a distracter appeared in the left or right hemifield for $300 \mathrm{~ms}$, followed by a blank screen (100 ms). The target display then appeared for 2000 ms. Participants were instructed to press a button with their right hand when they detected the target-colour in the centre, irrespective of its shape. We used only central targets in order to minimize reflexive glances to the periphery (and thus to minimize eye movement artifacts in EEG), and it was emphasized that subjects should never react to the peripheral distracter. There were 64 trials in each experiment block, one third of which contained a target (go trials) and the remaining a non-target (no-go trials). There were four distracter conditions varying orthogonally within each block (Figure $1 \mathrm{~A})$ : high relevance-high saliency $(\mathrm{HrHs})$; high relevance-low saliency (HrLs); low relevance-high saliency (LrHs) and low relevance-low saliency (LrLs). Every participant completed at least twenty blocks for a total of 1280 trials. Fixation was checked visually during the practice runs and periodically during the experimental runs.

\section{Insert Figure 1 about here}

\subsection{Lesion mapping}

Lesion mapping was based on structural magnetic resonance imaging (MRI) in 10 patients and on a CT scan for patient N1 (in whom MRI was not indicated due to a pacemaker). The imaging protocol included axial FLAIR, T1- and T2-weighted acquisitions as well as diffusion weighted MRI with a slice resolution of $4 \mathrm{~mm}$, performed on a 3T Siemens scanner (Siemens Medical Solutions, Erlangen, Germany). Time of imaging (mean, 45.7 days post-injury) was 
sufficiently distant from injury to allow detecting a clear boundary between damaged and intact tissue.

Damaged regions were delineated directly as volume of interest (VOI) on the axial T2- or on the diffusion-weighted MRI scans using a graphic tablet and MRIcron (Rorden, Karnath, \& Bonilha, 2007). The patient MRI and the VOI were then normalized to standard space using SPM8 (www.fil.ion.ucl.ac.uk/spm). A lesion mask was used to reduce the contribution of damaged tissue during normalization (Brett, Leff, Rorden, \& Ashburner, 2001). The normalized VOIs were smoothed and projected onto the T1-template MRI scan from the Montreal Neurological Institute (MNI, http://www.bic.mni.mcgill.ca). Spatial normalization of the CT scan of patient N1 and its VOI was performed with the clinical toolbox (Rorden, Bonilha, Fridriksson, Bender, \& Karnath, 2012), which includes a CT template for older population (mean 65 years old) that matches the MNI template. Overlaps were performed in MNI-space using MRIcron (Rorden, et al., 2007).

\subsection{EEG acquisition and analysis}

\subsubsection{EEG data acquisition}

EEG was recorded with a 128-channel Biosemi ActiveTwo EEG system (Biosemi B.V., Amsterdam, Netherlands) at a sampling rate of $512 \mathrm{~Hz}$ during a resting condition in which subjects were awake with their eyes closed. Artefacts and periods with low vigilance were excluded by visual inspection of the data and four minutes of artefact-free data were recalculated against the average reference.

\subsubsection{Functional connectivity analysis}

Functional connectivity (FC) at the cortical source level was calculated in Matlab (The MathWorks Inc., Natick, USA) with NUTMEG (http://nutmeg.berkeley.edu; Dalal et al., 2011) and the FCM toolbox (Niebur, et al., 1991). The detailed analysis steps and their validation have been described previously (Guggisberg et al., 2011). Lead field potential with $10 \mathrm{~mm}$ grid spacing was obtained using the Boundary Element Method (BEM) based on the segmented grey matter of individual T1 MRI scans for ten patients and on the MNI template MRI for patient N1 and for the healthy controls. Measuring FC with EEG faces the problem of source activity reconstruction, in particular in the presence of structural lesions. However, combining high resolution EEG with modern inverse solution approaches and realistic head shape models results in spatially adequate source reconstructions (Benar \& Gotman, 2002; Michel et al., 2004). Clinical studies have shown excellent accuracy of electrical source 
localization with large brain lesions when individual head models were used (Brodbeck, Lascano, Spinelli, Seeck, \& Michel, 2009; Guggisberg, et al., 2011).

The EEG data was bandpass-filtered between 1 and $20 \mathrm{~Hz}$ and non-overlapping data segments of 1-s duration were tapered with a Hanning window and Fourier transformed. Fourier coefficients were then projected to grey matter voxels with an adaptive spatial filter (scalar minimum variance beamformer (Sekihara, Nagarajan, Poeppel, \& Marantz, 2004). The absolute imaginary part of coherence (IC (Nolte et al., 2004; Sekihara, Owen, Trisno, \& Nagarajan, 2011)) was calculated between all possible pairs of voxels during the four minutes of resting-state recording. IC represents lagged coherence, which is a non-directed FC metric that measures the correlation between two signals in the frequency domain and mainly depends on the phase consistency between the signals. The advantage of IC over coherence or other measures of FC is that it is not subject to biases arising from volume conduction or spatial leakage of the inverse solution (Sekihara, et al., 2011). IC was calculated for seven frequency bands between 1 and $20 \mathrm{~Hz}$ : delta $(1-3 \mathrm{~Hz})$, low theta $(4-5 \mathrm{~Hz})$, high theta $(6-7 \mathrm{~Hz})$, low alpha $(8-10 \mathrm{~Hz})$, high alpha $(11-12 \mathrm{~Hz})$, low beta $(13-16 \mathrm{~Hz})$ and high beta $(17-20 \mathrm{~Hz})$.

The mean IC was calculated between each voxel and a given region of interest (ROI), composed of a cluster of voxels (hereafter termed 'seed'). FC maps were then standardized to the mean value across all voxels by calculating Z-scores. Z-score maps were spatially normalized to the canonical MNI brain space with SPM8 (http://www.fil.ion.ucl.ac.uk/spm/software/spm8/). Lesions were masked during spatial normalization to avoid distortion. A whole brain map of normalized FC was computed for all frequency bands and each of five right-hemispheric ROls defined on the Automated Anatomical Labeling (AAL) atlas (Tzourio-Mazoyer et al., 2002): the superior temporal gyrus (rSTG), the inferior parietal cortex (rIPC; composed of the right angular and supramarginal gyrus), the superior parietal cortex (rSPC), the dorsolateral prefrontal cortex (rDLPC; composed of the superior and middle frontal gyrus), and the inferior prefrontal cortex (rIPC; composed of the inferior frontal gyrus, pars triangularis) (Figure 2).

Unpaired T-tests were applied with statistical non-parametric mapping (SnPM) to assess differences in IC magnitude between patients and controls, for all ROIs and frequency bands. Correction for multiple comparisons was performed using thresholding based on the cluster size distribution obtained after random permutation of original data (Singh, Barnes, \& Hillebrand, 2003). Finally, correlations between FC and the relevance index were computed using the mean FC between two selected ROIs. The value was obtained by extracting from a seed FC map the average value of the target ROI. We performed Pearson correlations as 
normality tests indicated that distribution of the relevance index did not violate the normality assumption (Shapiro-Francia test: $p=0.17$ ). Correlations between FC and Bells cancellation test were obtained using the same method. Finally, to verify the spatial specificity of ROI correlations between FC and the relevance index, we also computed voxel-wise correlations using the same FC maps.

Insert Figure 2 about here

\section{RESULTS}

\subsection{Behavioural results}

Given the main effect of relevance in controls compared to patients we focused behavioural and connectivity analyses on the effects of distracter relevance. To directly compare the effect of distracter relevance on target detection between groups, we computed a relevance index $(\mathrm{RI})$ based on the reaction time $(\mathrm{RT})$ data measured in the four experimental conditions, using the following formula:

$$
\mathrm{RI}=(\mathrm{LrHs}+\mathrm{LrLs}-\mathrm{HrHs}-\mathrm{HrLs}) /(\mathrm{HrHs}+\mathrm{HrLs}+\mathrm{LrHs}+\mathrm{LrLs})
$$

The relevance index reflects the extent to which the speed of target detection depends on distracter relevance irrespective of baseline processing time and distracter saliency. A positive index reflects a processing advantage for targets following a relevant distracter compared to an irrelevant distracter. In order to examine whether network connectivity was a specific predictor of the relevance index, we also performed correlation analyses between FC and a saliency index $(\mathrm{SI}=(\mathrm{HrLs}+\mathrm{LrLs}-\mathrm{HrHs}-\mathrm{LrHs}) /(\mathrm{HrHs}+\mathrm{HrLs}+\mathrm{LrHs}+\mathrm{LrLs}))$, as well as between FC and performance on the Bells cancellation test (Gauthier, et al., 1989) (difference between omissions on the left side relative to the right side of space).

Figure 3 shows reaction time (RT) data of the two groups. In an initial analysis, a mixed ANOVA with between-factors group (healthy controls, patients) and within-factors visual field (left, right), distracter relevance (relevant, irrelevant) and distracter saliency (high, low) was computed. This analysis revealed significant main effects of group $(F(1,25)=12.2, p<0.01$, $\left.\eta_{\mathrm{p} 2}=.33\right)$ and saliency $\left(F(1,25)=4.3, \mathrm{p}<0.05, \eta_{\mathrm{p} 2}=.15\right)$. The interaction between group $\mathrm{X}$ visual field $\left(F(1,25)=7.1, p<0.05, \eta_{p 2}=.22\right)$ and the three-way interaction between visual field $X$ relevance $X$ saliency $\left(F(1,25)=6.3, p<0.05, \eta_{p 2}=.20\right)$ were also significant. Finally, the four-way interaction group $X$ visual field $X$ relevance $X$ saliency $(F(1,25)=5.8, p<0.05$, $\left.\eta_{\mathrm{p} 2}=.19\right)$ reached significance. 
Given the significant main effect of group and the four-way interaction including this factor, the data were followed up with separate analyses for each group. The data of healthy participants were only characterized by a significant main effect of distracter relevance $(F$ $\left.(1,15)=11.1 \mathrm{p}<0.01, \eta_{\mathrm{p} 2}=.43\right)$ reflecting faster reactions with high-relevance compared to low-relevance distracters, irrespective of the visual field and saliency. In contrast, the patients group showed a significant main effect of visual field $\left(F(1,10)=5.3, p<0.05, \eta_{p 2}=\right.$ .35), while the three-way interaction between visual field $X$ relevance $X$ saliency approached significance $\left(F(1,10)=4.9, p=0.052, \eta_{p 2}=.33\right)$. Further analyses of this interaction effect with post-hoc Bonferroni tests revealed that distracters with low saliency and low relevance led to increased target detection time when they were presented in the right visual field compared to the left visual field. Thus, patients didn't show the main effect of distracter relevance compared to controls, irrespective of their visual field of apparition. Omission rates (controls: $2.8 \% \pm 1.6$; patients: $5.5 \% \pm 2.1$ ) and false positive reactions (controls: $1.9 \% \pm 1.5$; patients: $2.1 \% \pm 1$ ) were low in both groups and were therefore not analysed further.

Finally, we found weak correlations between the relevance index and performance on the Bells cancellation task (left visual field: $r=0.54, p=0.08$; right visual field: $r=0.46, p=0.15$ ).

Insert Figure 3 about here

\subsection{Lesion-mapping results}

Figure 4 shows lesion overlap of patients. The greatest overlap was found in right subcortical white matter adjacent to the lateral ventricle. Regions within the dorsal fronto-parietal network (superior parietal cortex and dorsal prefrontal cortex) were generally spared.

Insert Figure 4 about here

\subsection{Functional connectivity results}

\subsubsection{Connectivity as predictor of between-group differences}

Among the frequency bands and ROIs analysed for connectivity, the most prominent differences observed between patients and healthy controls concerned the right superior parietal cortex and the right prefrontal cortex in the high theta band $(6-7 \mathrm{~Hz})$. A comparison of mean normalized theta band connectivity between groups revealed significantly higher 
functional connectivity in healthy controls than patients in the right dorsolateral prefrontal cortex (rDLPC) and the right superior parietal cortex (rSPC). The topographic maps in Figure 5 show the only significant voxels of the group comparison surviving correction among all ROIs and frequency bands examined. Note that connectivity was computed between the seed regions and the entire brain. When the rDLPC was set as seed region, patients showed reduced connectivity of an area centred on the superior parietal lobule and post-central gyrus (Figure 5A). When the rSPC was defined as seed, decreased connectivity was found in patients mainly in the right middle frontal gyrus and lateral orbital cortex (Figure 5B). The spectrogram plot in Figure 5C illustrates the theta-band specificity of the observed reduced FC network in patients compared to controls.

Insert Figure 5 about here

\subsubsection{Connectivity as predictor of within-group behavioural performance}

Based on the significant difference of theta-band FC maps between groups, we computed the mean functional connectivity between the rSPC and the rDLPC and correlated it with the relevance index (Figure 6). In the control group, the correlation was significant for distracters in the left visual field $(p<0.05)$ and marginally significant for distracters in the right visual field $(p=0.055$ ) (Figure 6A and 6B). In patients, the correlation reached significance for both visual fields ( $p<0.05$; Figure $6 C$ and 6D). Importantly, the rSPC-rDLPC FC did not correlate significantly with lesion volume $(r=-0.12, p=0.73)$. In addition, a partial correlation analysis indicated that the correlations between rSPC-rDLPC connectivity and relevance index were not related to lesion volume (left visual field: $r=0.66, p=0.04$; right visual field: $r=0.70, p=$ 0.02). Because of the small size of our samples we performed permutation tests to confirm the significance of our correlation results. Combining the results of both groups, we found significant results for correlations between dorsal fronto-parietal FC and the relevance index for distracters shown in the left $(p=0.003)$ and in the right visual field $(p=0.008)$. When permutation tests were applied for each group separately, the analysis confirmed in patients a significant correlation between dorsal fronto-parietal FC and the relevance index for distracters in the right visual field $(r=0.7, p=0.03)$, while there was only a trend for the left visual field $(r=0.7, p=0.09)$. In healthy controls, marginally significant results were obtained for the left $(r=0.5, p=0.05)$ and right visual field $(r=0.5, p=0.055)$.

Insert Figure 6 about here 
To verify the spatial specificity of these findings, we also correlated normalized functional connectivity in each voxel with the relevance index. Figure 7 shows for each group voxelwise correlation maps with the rDLPC and the rSPC as seed regions. Both groups showed similar correlation patterns within the right-hemispheric dorsal fronto-parietal cortex, whether the relevance effect was based on distracters presented in the left or the right visual field.

Insert Figure 7 about here

Finally, to verify the particular importance of dorsal fronto-parietal connectivity for the relevance index (top-down attention), we also computed correlations between rSPC-rDLPC connectivity and the saliency index. None of these correlations were significant (Figure 8; controls: left visual field $r=-0.32, p=0.23$, right visual field: $r=0.06 ; p=0.81$; patients: left visual field $r=-0.41, p=0.21$, right visual field $r=0.21, p=0.53$ ). Finally, the correlation between rSPC-rDLPC connectivity and performance on the Bells cancellation test did not reach significance $(p=0.67)$.

Insert Figure 8 about here

\section{DISCUSSION}

Goal-directed attention denotes a series of factors whose collective effect is to bias sensory processing according to behavioural goals (Tsotsos, 2011). A controversy concerns the degree of functional preservation of these factors in brain-damaged patients with deficits of spatial attention (Aglioti, et al., 1997; Esterman, et al., 2000; Fellrath, et al., 2012; Ptak \& Golay, 2006; Ptak \& Schnider, 2006; Riddoch \& Humphreys, 1987; Snow \& Mattingley, 2006). Our findings demonstrate that impaired goal-directed attention is associated with reduced theta-band connectivity in the dorsal fronto-parietal network. These observations are related to the findings of previous fMRI studies reporting abnormal blood-oxygen patterns associated with top-down control of attention in spatial neglect (Baldassarre, et al., 2014; He, et al., 2007), and bring complementary information about the implication of the theta-band frequency.

\subsection{Spatially lateralized and non-lateralized impairments of attention}

Our study aimed to investigate the capture of attention by stimuli whose relevance to the task was determined by their colour. Importantly, participants never reacted to the distracters, 
their impact on target processing therefore only being due to the contingent relationship between distracter features and target features. The control group showed perceptual facilitation of target detection by task-relevant compared to irrelevant distracters. While previous studies attributed facilitation to feature priming (Theeuwes, 2013), several observations suggest that in our task it was due to the attentional set of observers. First, distracters and targets were never displayed at the same location, reducing potential "positional priming" effects. Second, in a previous study using this task (Fellrath, et al., 2014), distracter processing was associated with distinct processing stages, starting with pure effects of stimulus saliency and ending with pure relevance effects (>180 ms). These results agree with the view that early attention effects are primarily driven by perceptual characteristics of the stimulus during fast feedforward processing, while top-down factors influence processing at later stages (Lamme \& Roelfsema, 2000; Theeuwes, 2010). Importantly, only the late relevance effect was a predictor of performance in the task. Finally, theta-band FC is particularly important for working memory processes (de Araujo, Baffa, \& Wakai, 2002; Tesche \& Karhu, 2000), which in our study might reflect the task requirement to actively hold a target colour in memory. Thus, a possible mechanism explaining facilitation effects in our task is top-down attentional guidance of target processing by the target-defining features of the distracter (e.g. 'redness' when the target was red; Wolfe, 1994; Wolfe \& Horowitz, 2004). According to this account, attending to target features (even involuntarily) may activate the corresponding attentional template in working memory, thereby enhancing the identification of a matching stimulus (Moore \& Weissman, 2010). This process didn't appear to influence performance of patients, who didn't show significant facilitation by distracter relevance, regardless of their side of apparition. This observation suggests a nonlateralized impairment of goal-directed processing of peripheral stimuli with target-defining features. Besides, consistent with their lateralized spatial deficits, patients had slower RTs following ipsilesional than contralesional distracters. This finding suggests an exaggerated capture of attention by ipsilesional stimuli, one of the predominant signs of the neglect syndrome (Gainotti, D'Erme, \& Bartolomeo, 1991; Kinsbourne, 1987; Mark, Kooistra, \& Heilman, 1988; Marshall \& Halligan, 1988; Schnider, Durbec, \& Ptak, 2011).

\subsection{Dorsal fronto-parietal network and goal-directed attention}

Functional neuroimaging studies have shown that feature-based attention activates similar areas in the dorsal fronto-parietal network as spatial attention (Giesbrecht, Woldorff, Song, \& Mangun, 2003; Slagter et al., 2007; Wojciulik \& Kanwisher, 1999), supporting the involvement of a domain-general attentional control mechanism. This is also supported by an event-related potential study of healthy participants performing the present visuo-spatial task (Fellrath, et al., 2014). Although in that study saliency effects (>70 ms) and relevance effects 
(>180 ms) dissociated in time, they shared a similar source of activity in the right intraparietal sulcus and more anterior regions of the fronto-parietal convexity. Thus, the task has the advantage to measure early, involuntary effects of feature relevance on attentional selection that rely on the fronto-parietal attention network. The absence of such effects in patients of the present study therefore suggests a functional impairment of the right dorsal frontoparietal network. This is consistent with our analyses of functional connectivity. We observed a significant decrease of theta-band functional connectivity between the right dorsolateral prefrontal cortex and the right superior parietal region in patients compared to controls. In addition, a significant correlation between connectivity and the behavioural impact of distracter relevance emerged: the stronger the theta-band functional connectivity between the right dorsolateral prefrontal cortex and the right superior parietal region the more was RT influenced by distracter relevance. Permutation tests suggested that this finding concerned both groups, though some of the within-group comparisons only approached significance. The latter findings should therefore be interpreted with caution as the group size was at the lower limit to justify within-group comparisons. Nevertheless, the relation between connectivity and distracter relevance contrasts with an absence of such a relation with the behavioural impact of saliency or the performance on a classic cancellation test used to assess exploratory behaviour in neglect (the Bells test; Gauthier, et al., 1989). The present findings therefore demonstrate the importance and specificity of dorsal fronto-parietal connectivity with goal-directed, feature-based attention.

Our results are in line with previous studies supporting a central role of the dorsal frontoparietal network in attentional control and guidance based on behavioural goals. Single-cell studies in the monkey and human neuroimaging studies have shown that dorsolateral prefrontal cortex and intraparietal cortex enhance stimulus and object attributes when attention is deployed based on feature cues (e.g., 'search for red') or locational cues (e.g., 'search in the upper right corner') (Bisley \& Goldberg, 2010; Caspari, Janssens, Mantini, Vandenberghe, \& Vanduffel, 2015; Scolari, Seidl-Rathkopf, \& Kastner, 2015). Attention in these studies describes the covert selection of relevant over irrelevant stimuli based on unique features or specific spatial coordinates (Duncan, 1984). The critical structures in the frontal lobes appear to be the dorsal prefrontal and premotor cortices, in particular the frontal eye field (FEF). Dorsolateral prefrontal cortex has been associated with executive control (Miller \& Cohen, 2001) and working memory for locations and objects (Everling, Tinsley, Gaffan, \& Duncan, 2006; Miller, Erickson, \& Desimone, 1996; Rainer, Asaad, \& Miller, 1998; Rao, Rainer, \& Miller, 1997), while the FEF has a decisive role for voluntary saccade planning and spatial attention (Moore \& Fallah, 2001). The posterior node of the dorsal fronto-parietal attention network is the lateral intraparietal area, which contains neurons that 
selectively code combinations of features when these become task-relevant (Ibos \& Freedman, 2014; Siegel, Buschman, \& Miller, 2015). Given that task-related modulation of attention represents a mechanism that controls which stimulus is selected, it is important to understand the conditions favouring prefrontal and parietal cortex interactions to achieve such control.

The importance of direct fronto-parietal connectivity for top-down attention is documented in structural connectivity studies. For example, diffusion-weighted imaging of the superior longitudinal fasciculus - the main fibre tract connecting posterior parietal with dorsal premotor cortex - suggests that the efficiency of attentional selection in visuospatial tasks is related to the strength of fronto-parietal connections (Thiebaut de Schotten et al., 2011; Tuch et al., 2005). In addition, the degree of preservation of these fibres in spatial neglect determines to what extent patients will attend to task-relevant stimulus features (Ptak \& Schnider, 2010). EEG FC complements these structural connectivity findings with measures of oscillatory dynamics and frequency-specific coupling across brain regions (Honey \& Sporns, 2008; Siegel, Donner, \& Engel, 2012). This approach does not require knowing the precise cause underlying reduced FC (i.e., whether it is a disconnecting lesion or a cortical lesion affecting FC directly or indirectly), and it is therefore important to compute FC irrespective of the presence of structural tissue damage. The fact that FC was not affected comparably across different oscillation frequencies and did not correlate with lesion volume suggests that our approach captured residual function of damaged tissue. Note however, that due to the absence of a brain-injured control group our findings do not allow making a direct link between decreased FC and spatial neglect. Rather, the observed changes in functional network organization indicate that intrahemispheric fronto-parietal disconnection is crucial for the occurrence of goal-directed attention deficits following right brain damage.

\subsection{The role of theta-frequency band for attentive processing}

Our results revealed consistent predictive power of fronto-parietal phase synchrony connectivity in a specific oscillatory frequency - the theta band. Recent electrophysiological studies have shown that top-down attentional selection is dependent on long-range phasesynchronization at narrow-band frequencies (Fries, 2005; Siegel, et al., 2012). Several studies indicate that theta frequencies in the frontal brain regions predict the amount of information actively maintained in working memory (de Araujo, et al., 2002; Tesche \& Karhu, 2000) and is associated with attentional control processes (Hegde \& Felleman, 1999). These observations fit well with the observed association of impaired reorienting toward contingent distracters and decreased functional connectivity in the theta frequency range. They also agree with our observation that the spatial distribution of theta-band functional connectivity 
reflects the involvement of top-down attention in a spatial attention task. Together, these findings suggest that long-range integration of goal-relevant information across the dorsal fronto-parietal network is coordinated through theta frequency coherence. However, this finding should not be interpreted as contradicting the importance of other frequency bands. Other studies demonstrated that performance on cognitive tasks is linearly associated with the magnitude of resting-state coherence in the alpha band (Dubovik et al., 2013; Rizk, Ptak, Nyffeler, Schnider, \& Guggisberg, 2013). In particular, several studies have found correlations between alpha band FC and traditional neglect tests (Sasaki et al., 2013), visual exploration (Rizk, et al., 2013), or spatial working memory (Dubovik, et al., 2012). These observations suggest that different cognitive functions rely on distinct EEG rhythms (Klimesch, Freunberger, Sauseng, \& Gruber, 2008) and that the relationship between theta band FC and the modulation of attention by relevance rely on a specific neural mechanism involving the dorsal fronto-parietal network.

In sum, the present study provides clues about the contribution of a dorsal fronto-parietal network to specific attentional capacities. First, a disruption of cortical resting-state functional connectivity in the theta frequency range between core regions of the dorsal fronto-parietal attention network - the superior parietal and dorsolateral prefrontal cortex is observed in right brain damaged patients with spatial attention deficits. Second, changes in theta-band range within this brain network are associated with impaired feature-based top-down attention. Resting-state EEG analyses provide the opportunity to relate behavioural deficits of patients with focal brain damage to impaired function of specific brain networks.

\section{ACKNOWLEDGEMENTS}

Study supported by Swiss National Science Foundation grants 320030-152689 to R. Ptak and 320030-146639 to A. G. Guggisberg. 


\section{REFERENCES}

Aglioti, S., Smania, N., Barbieri, C., \& Corbetta, M. (1997). Influence of stimulus salience and attentional demands on visual search patterns in hemispatial neglect. Brain and Cognition, 34(3), 388-403.

Alstott, J., Breakspear, M., Hagmann, P., Cammoun, L., \& Sporns, O. (2009). Modeling the impact of lesions in the human brain. PLoS Comput Biol, 5(6), e1000408.

Baldassarre, A., Ramsey, L., Hacker, C. L., Callejas, A., Astafiev, S. V., Metcalf, N. V., et al. (2014). Large-scale changes in network interactions as a physiological signature of spatial neglect. Brain, 137(Pt 12), 3267-3283.

Bartolomeo, P., Sieroff, E., Decaix, C., \& Chokron, S. (2001). Modulating the attentional bias in unilateral neglect: the effects of the strategic set. Exp Brain Res, 137(3-4), 432-444.

Benar, C. G., \& Gotman, J. (2002). Modeling of post-surgical brain and skull defects in the EEG inverse problem with the boundary element method. Clin Neurophysiol, 113(1), 48-56.

Bisley, J. W., \& Goldberg, M. E. (2010). Attention, intention, and priority in the parietal lobe. Annu Rev Neurosci, 33, 1-21.

Brett, M., Leff, A. P., Rorden, C., \& Ashburner, J. (2001). Spatial normalization of brain images with focal lesions using cost function masking. Neuroimage, 14(2), 486-500.

Brodbeck, V., Lascano, A. M., Spinelli, L., Seeck, M., \& Michel, C. M. (2009). Accuracy of EEG source imaging of epileptic spikes in patients with large brain lesions. Clin Neurophysiol, 120(4), 679685.

Caspari, N., Janssens, T., Mantini, D., Vandenberghe, R., \& Vanduffel, W. (2015). Covert shifts of spatial attention in the macaque monkey. J Neurosci, 35(20), 7695-7714.

Committeri, G., Pitzalis, S., Galati, G., Patria, F., Pelle, G., Sabatini, U., et al. (2007). Neural bases of personal and extrapersonal neglect in humans. Brain, 130(Pt 2), 431-441.

Corbetta, M., Kincade, M. J., Lewis, C., Snyder, A. Z., \& Sapir, A. (2005). Neural basis and recovery of spatial attention deficits in spatial neglect. Nat Neurosci, 8(11), 1603-1610.

Corbetta, M., \& Shulman, G. L. (2002). Control of goal-directed and stimulus-driven attention in the brain. Nat Rev Neurosci, 3(3), 201-215.

Dalal, S. S., Zumer, J. M., Guggisberg, A. G., Trumpis, M., Wong, D. D., Sekihara, K., et al. (2011). MEG/EEG source reconstruction, statistical evaluation, and visualization with NUTMEG. Comput Intell Neurosci, 2011, 758973.

de Araujo, D. B., Baffa, O., \& Wakai, R. T. (2002). Theta oscillations and human navigation: a magnetoencephalography study. J Cogn Neurosci, 14(1), 70-78.

Dubovik, S., Pignat, J. M., Ptak, R., Aboulafia, T., Allet, L., Gillabert, N., et al. (2012). The behavioral significance of coherent resting-state oscillations after stroke. Neuroimage, 61(1), 249-257.

Dubovik, S., Ptak, R., Aboulafia, T., Magnin, C., Gillabert, N., Allet, L., et al. (2013). EEG alpha band synchrony predicts cognitive and motor performance in patients with ischemic stroke. Behav Neurol, 26(3), 187-189.

Duncan, J. (1984). Selective attention and the organization of visual information. J Exp Psychol Gen, 113(4), 501-517.

Esterman, M., McGlinchey-Berroth, R., \& Milberg, W. (2000). Preattentive and attentive visual search in individuals with hemispatial neglect. Neuropsychology, 14(4), 599-611.

Everling, S., Tinsley, C. J., Gaffan, D., \& Duncan, J. (2006). Selective representation of task-relevant objects and locations in the monkey prefrontal cortex. Eur J Neurosci, 23(8), 2197-2214.

Fellrath, J., Blanche-Durbec, V., Schnider, A., Jacquemoud, A. S., \& Ptak, R. (2012). Visual search in spatial neglect studied with a preview paradigm. Front Hum Neurosci, 6, 93.

Fellrath, J., Manuel, A. L., \& Ptak, R. (2014). Task relevance effects in electrophysiological brain activity: early, but not first. Neuroimage, 101, 68-75.

Fries, P. (2005). A mechanism for cognitive dynamics: neuronal communication through neuronal coherence. Trends Cogn Sci, 9(10), 474-480. 
Gainotti, G., D'Erme, P., \& Bartolomeo, P. (1991). Early orientation of attention toward the half space ipsilateral to the lesion in patients with unilateral brain damage. $J$ Neurol Neurosurg Psychiatry, 54(12), 1082-1089.

Gainotti, G., Messerli, P., \& Tissot, R. (1972). Qualitative analysis of unilateral spatial neglect in relation to laterality of cerebral lesions. J Neurol Neurosurg Psychiatry, 35(4), 545-550.

Gauthier, L., Dehaut, F., \& Joanette, Y. (1989). The Bells Test - a Quantitative and Qualitative Test for Visual Neglect. International Journal of Clinical Neuropsychology, 11(2), 49-54.

Giesbrecht, B., Woldorff, M. G., Song, A. W., \& Mangun, G. R. (2003). Neural mechanisms of topdown control during spatial and feature attention. Neuroimage, 19(3), 496-512.

Golay, L., Schnider, A., \& Ptak, R. (2008). Cortical and subcortical anatomy of chronic spatial neglect following vascular damage. Behav Brain Funct, 4, 43.

Grandjean, D., Sander, D., Lucas, N., Scherer, K. R., \& Vuilleumier, P. (2008). Effects of emotional prosody on auditory extinction for voices in patients with spatial neglect. Neuropsychologia, 46(2), 487-496.

Guggisberg, A. G., Dalal, S. S., Zumer, J. M., Wong, D. D., Dubovik, S., Michel, C. M., et al. (2011). Localization of cortico-peripheral coherence with electroencephalography. Neuroimage, 57(4), 1348-1357.

Guggisberg, A. G., Honma, S. M., Findlay, A. M., Dalal, S. S., Kirsch, H. E., Berger, M. S., et al. (2008). Mapping functional connectivity in patients with brain lesions. Annals of Neurology, 63(2), 193-203.

Halligan, P. W., Fink, G. R., Marshall, J. C., \& Vallar, G. (2003). Spatial cognition: evidence from visual neglect. Trends Cogn Sci, 7(3), 125-133.

He, B. J., Snyder, A. Z., Vincent, J. L., Epstein, A., Shulman, G. L., \& Corbetta, M. (2007). Breakdown of functional connectivity in frontoparietal networks underlies behavioral deficits in spatial neglect. Neuron, 53(6), 905-918.

Hegde, J., \& Felleman, D. J. (1999). The popout in some conjunction searches is due to perceptual grouping. Neuroreport, 10(1), 143-148.

Heilman, K., Watson, R., \& Valenstein, E. (1993). Neglect and related disorders. In E. V. KM Heilman (Ed.), Clinical Neuropsychology (pp. 279-336). New-York : Oxford UP.

Honey, C. J., \& Sporns, O. (2008). Dynamical consequences of lesions in cortical networks. Hum Brain Mapp, 29(7), 802-809.

Ibos, G., \& Freedman, D. J. (2014). Dynamic integration of task-relevant visual features in posterior parietal cortex. Neuron, 83(6), 1468-1480.

Karnath, H. O., Berger, M. F., Kuker, W., \& Rorden, C. (2004). The anatomy of spatial neglect based on voxelwise statistical analysis: A study of 140 patients. Cereb Cortex, 14(10), 1164-1172.

Karnath, H. O., Ferber, S., \& Himmelbach, M. (2001). Spatial awareness is a function of the temporal not the posterior parietal lobe. Nature, 411(6840), 950-953.

Karnath, H. O., Himmelbach, M., \& Kuker, W. (2003). The cortical substrate of visual extinction. Neuroreport, 14(3), 437-442.

Kinsbourne, M. (1987). Mechanisms of unilateral negelct: evidence from attentional gradients within hemispace. In M. Jeannerod (Ed.), Neurophysiological and Neuropsychological Aspects of Spatial Neglect (pp. 69-86). Amsterdam: North-Holland.

Klimesch, W., Freunberger, R., Sauseng, P., \& Gruber, W. (2008). A short review of slow phase synchronization and memory: evidence for control processes in different memory systems? Brain Res, 1235, 31-44.

Koyama, M. S., Di Martino, A., Zuo, X. N., Kelly, C., Mennes, M., Jutagir, D. R., et al. (2011). Restingstate functional connectivity indexes reading competence in children and adults. J Neurosci, 31(23), 8617-8624.

Lamme, V. A., \& Roelfsema, P. R. (2000). The distinct modes of vision offered by feedforward and recurrent processing. Trends Neurosci, 23(11), 571-579.

Mannan, S. K., Kennard, C., \& Husain, M. (2009). The role of visual salience in directing eye movements in visual object agnosia. Curr Biol, 19(6), R247-248. 
Mark, V. W., Kooistra, C. A., \& Heilman, K. M. (1988). Hemispatial neglect affected by non-neglected stimuli. Neurology, 38(8), 1207-1211.

Marshall, J. C., \& Halligan, P. W. (1988). Blindsight and insight in visuo-spatial neglect. Nature, 336(6201), 766-767.

Martino, J., Honma, S. M., Findlay, A. M., Guggisberg, A. G., Owen, J. P., Kirsch, H. E., et al. (2011). Resting functional connectivity in patients with brain tumors in eloquent areas. Annals of Neurology, 69(3), 521-532.

Michel, C. M., Murray, M. M., Lantz, G., Gonzalez, S., Spinelli, L., \& Grave de Peralta, R. (2004). EEG source imaging. Clin Neurophysiol, 115(10), 2195-2222.

Miller, E. K., \& Cohen, J. D. (2001). An integrative theory of prefrontal cortex function. Annu Rev Neurosci, 24, 167-202.

Miller, E. K., Erickson, C. A., \& Desimone, R. (1996). Neural mechanisms of visual working memory in prefrontal cortex of the macaque. Journal of Neuroscience, 16(16), 5154-5167.

Moore, K. S., \& Weissman, D. H. (2010). Involuntary transfer of a top-down attentional set into the focus of attention: Evidence from a contingent attentional capture paradigm. Attention Perception \& Psychophysics, 72(6), 1495-1509.

Moore, T., \& Fallah, M. (2001). Control of eye movements and spatial attention. Proc Natl Acad Sci U $S A, 98(3), 1273-1276$.

Mort, D. J., Malhotra, P., Mannan, S. K., Rorden, C., Pambakian, A., Kennard, C., et al. (2003). The anatomy of visual neglect. Brain, 126(Pt 9), 1986-1997.

Natale, E., Posteraro, L., Prior, M., \& Marzi, C. A. (2005). What kind of visual spatial attention is impaired in neglect? Neuropsychologia, 43(7), 1072-1085.

Nicolo, P., Rizk, S., Magnin, C., Pietro, M. D., Schnider, A., \& Guggisberg, A. G. (2015). Coherent neural oscillations predict future motor and language improvement after stroke. Brain, 138(Pt 10), 3048-3060.

Niebur, E., Schuster, H. G., Kammen, D. M., \& Koch, C. (1991). Oscillator-phase coupling for different two-dimensional network connectivities. Phys Rev A, 44(10), 6895-6904.

Nolte, G., Bai, O., Wheaton, L., Mari, Z., Vorbach, S., \& Hallett, M. (2004). Identifying true brain interaction from EEG data using the imaginary part of coherency. Clinical Neurophysiology, 115(10), 2292-2307.

Oxbury, J. M., Campbell, D. C., \& Oxbury, S. M. (1974). Unilateral spatial neglect and impairments of spatial analysis and visual perception. Brain, 97(3), 551-564.

Ptak, R., Di Pietro, M., \& Schnider, A. (2012). The neural correlates of object-centered processing in reading: a lesion study of neglect dyslexia. Neuropsychologia, 50(6), 1142-1150.

Ptak, R., \& Golay, L. (2006). Temporal dynamics of attentional control settings in patients with spatial neglect. Brain Res, 1092(1), 190-197.

Ptak, R., \& Schnider, A. (2006). Reflexive orienting in spatial neglect is biased towards behaviourally salient stimuli. Cereb Cortex, 16(3), 337-345.

Ptak, R., \& Schnider, A. (2010). The dorsal attention network mediates orienting toward behaviorally relevant stimuli in spatial neglect. J Neurosci, 30(38), 12557-12565.

Ptak, R., Schnider, A., Golay, L., \& Muri, R. (2007). A non-spatial bias favouring fixated stimuli revealed in patients with spatial neglect. Brain, 130(Pt 12), 3211-3222.

Rainer, G., Asaad, W. F., \& Miller, E. K. (1998). Selective representation of relevant information by neurons in the primate prefrontal cortex. Nature, 393(6685), 577-579.

Rao, S. C., Rainer, G., \& Miller, E. K. (1997). Integration of what and where in the primate prefrontal cortex. Science, 276(5313), 821-824.

Riddoch, M., \& Humphreys, G. (1987). Perceptual and action systems in unilateral neglect. In M. Jeannerod (Ed.), Neurophysiological and Neuropsychological Aspects of Spatial Neglect (pp. 151-181). North-Holland: Elsevier.

Rizk, S., Ptak, R., Nyffeler, T., Schnider, A., \& Guggisberg, A. G. (2013). Network mechanisms of responsiveness to continuous theta-burst stimulation. Eur J Neurosci, 38(8), 3230-3238. 
Ronchi, R., Posteraro, L., Fortis, P., Bricolo, E., \& Vallar, G. (2009). Perseveration in left spatial neglect: drawing and cancellation tasks. Cortex, 45(3), 300-312.

Rorden, C., Bonilha, L., Fridriksson, J., Bender, B., \& Karnath, H. O. (2012). Age-specific CT and MRI templates for spatial normalization. Neuroimage, 61(4), 957-965.

Rorden, C., Karnath, H. O., \& Bonilha, L. (2007). Improving lesion-symptom mapping. J Cogn Neurosci, 19(7), 1081-1088.

Samuelsson, H., Jensen, C., Ekholm, S., Naver, H., \& Blomstrand, C. (1997). Anatomical and neurological correlates of acute and chronic visuospatial neglect following right hemisphere stroke. Cortex, 33(2), 271-285.

Sasaki, T., Abe, M., Okumura, E., Okada, T., Kondo, K., Sekihara, K., et al. (2013). Disturbed resting functional inter-hemispherical connectivity of the ventral attentional network in alpha band is associated with unilateral spatial neglect. PLoS One, 8(9), e73416.

Schnider, A., Durbec, V. B., \& Ptak, R. (2011). Absence of visual feedback abolishes expression of hemispatial neglect in self-guided spatial completion. J Neurol Neurosurg Psychiatry, 82(11), $1279-1282$.

Schoffelen, J. M., \& Gross, J. (2009). Source connectivity analysis with MEG and EEG. Hum Brain Mapp, 30(6), 1857-1865.

Scolari, M., Seidl-Rathkopf, K., \& Kastner, S. (2015). Functions of the human frontoparietal attention network: Evidence from neuroimaging. Current Opinion in Behavioral Sciences., Volume 1, 32-39.

Sekihara, K., Nagarajan, S. S., Poeppel, D., \& Marantz, A. (2004). Asymptotic SNR of scalar and vector minimum-variance beamformers for neuromagnetic source reconstruction. IEEE Trans Biomed Eng, 51(10), 1726-1734.

Sekihara, K., Owen, J. P., Trisno, S., \& Nagarajan, S. S. (2011). Removal of spurious coherence in MEG source-space coherence analysis. IEEE Trans Biomed Eng, 58(11), 3121-3129.

Siegel, M., Buschman, T. J., \& Miller, E. K. (2015). Cortical information flow during flexible sensorimotor decisions. Science, 19(348 (6241)), 1352-1355.

Siegel, M., Donner, T. H., \& Engel, A. K. (2012). Spectral fingerprints of large-scale neuronal interactions. [10.1038/nrn3137]. Nat Rev Neurosci, 13(2), 121-134.

Singh, K. D., Barnes, G. R., \& Hillebrand, A. (2003). Group imaging of task-related changes in cortical synchronisation using nonparametric permutation testing. Neuroimage, 19(4), 1589-1601.

Slagter, H. A., Giesbrecht, B., Kok, A., Weissman, D. H., Kenemans, J. L., Woldorff, M. G., et al. (2007). $\mathrm{fMRI}$ evidence for both generalized and specialized components of attentional control. Brain Res, 1177, 90-102.

Snow, J. C., \& Mattingley, J. B. (2006). Goal-driven selective attention in patients with right hemisphere lesions: how intact is the ipsilesional field? Brain, 129, 168-181.

Tesche, C. D., \& Karhu, J. (2000). Theta oscillations index human hippocampal activation during a working memory task. Proc Natl Acad Sci U S A, 97(2), 919-924.

Theeuwes, J. (2010). Top-down and bottom-up control of visual selection. Acta Psychol (Amst), 135(2), 77-99.

Theeuwes, J. (2013). Feature-based attention: it is all bottom-up priming. Philos Trans $R$ Soc Lond $B$ Biol Sci, 368(1628), 20130055.

Thiebaut de Schotten, M., Dell'Acqua, F., Forkel, S. J., Simmons, A., Vergani, F., Murphy, D. G. M., et al. (2011). A lateralized brain network for visuospatial attention. Nat Neurosci, 14(10), 12451246.

Ticini, L. F., de Haan, B., Klose, U., Nagele, T., \& Karnath, H. O. (2010). The role of temporo-parietal cortex in subcortical visual extinction. J Cogn Neurosci, 22(9), 2141-2150.

Tsotsos, J. K. (2011). A computational perspective on visual attention. The MIT Press.

Tuch, D. S., Salat, D. H., Wisco, J. J., Zaleta, A. K., Hevelone, N. D., \& Rosas, H. D. (2005). Choice reaction time performance correlates with diffusion anisotropy in white matter pathways supporting visuospatial attention. Proc Natl Acad Sci U S A, 102(34), 12212-12217. 
Tzourio-Mazoyer, N., Landeau, B., Papathanassiou, D., Crivello, F., Etard, O., Delcroix, N., et al. (2002). Automated anatomical labeling of activations in SPM using a macroscopic anatomical parcellation of the MNI MRI single-subject brain. Neuroimage, 15(1), 273-289.

Vallar, G., \& Perani, D. (1986). The anatomy of unilateral neglect after right-hemisphere stroke lesions. A clinical/CT-scan correlation study in man. Neuropsychologia, 24(5), 609-622.

Varela, F., Lachaux, J. P., Rodriguez, E., \& Martinerie, J. (2001). The brainweb: phase synchronization and large-scale integration. Nat Rev Neurosci, 2(4), 229-239.

Vossel, S., Eschenbeck, P., Weiss, P. H., Weidner, R., Saliger, J., Karbe, H., et al. (2011). Visual extinction in relation to visuospatial neglect after right-hemispheric stroke: quantitative assessment and statistical lesion-symptom mapping. J Neurol Neurosurg Psychiatry, 82(8), 862-868.

Wang, L., Negreira, A., LaViolette, P., Bakkour, A., Sperling, R. A., \& Dickerson, B. C. (2010). Intrinsic interhemispheric hippocampal functional connectivity predicts individual differences in memory performance ability. Hippocampus, 20(3), 345-351.

Weintraub, S., \& Mesulam, M. M. (1987). Right cerebral dominance in spatial attention. Further evidence based on ipsilateral neglect. Arch Neurol, 44(6), 621-625.

Wojciulik, E., \& Kanwisher, N. (1999). The generality of parietal involvement in visual attention. Neuron, 23(4), 747-764.

Wolfe, J. M. (1994). Guided Search 2.0 A revised model of visual search. Psychon Bull Rev, 1(2), 202238.

Wolfe, J. M., \& Horowitz, T. S. (2004). What attributes guide the deployment of visual attention and how do they do it? Nat Rev Neurosci, 5(6), 495-501.

Zhao, J., Liu, J., Li, J., Liang, J., Feng, L., Ai, L., et al. (2011). Intrinsically organized network for word processing during the resting state. Neurosci Lett, 487(1), 27-31. 


\section{Figure legends}

Figure 1. A) Example of stimuli used in the experiment. In the condition shown the target and relevant distracters were blue while irrelevant distracters were red or green (relevance modulation). Distracters in each relevance condition either had low or high luminance contrast with the background (saliency modulation). B) Time-course of events presented in one experiment trial. The example shows a trial with an irrelevant, high saliency distracter and a blue target. Note that in reality all stimuli were shown on neutral grey background and that the size of the stimuli is exaggerated.

Figure 2. 3D projection of ROIs. A) Right dorsolateral prefrontal cortex B) Right superior parietal cortex C) Right inferior parietal cortex D) Right superior temporal gyrus E) Right inferior prefrontal cortex.

Figure 3. Reaction time data as a function of group (controls, patients), distracter condition (high/low relevance; high/low saliency) and the visual field of apparition of the distracter (LVF, left; RVF, right visual field). Mean reaction time and standard deviations in the control group for distracters in the left visual field: HrHs (515.3 \pm 68.8$)$, HrLs (515.5 \pm 64.9$)$, LrHs (533.9 \pm 78.1$)$, LrLs (539.4 \pm 81.1$)$; and in the right visual field: HrHs (515.9 \pm 80.7$)$, HrLs (517.3 \pm 72.1$)$, LrHs (531.6 \pm 86.8$)$, LrLs (538.9 \pm 83$)$. Mean reaction time and standard deviations in the group of patients for distracters in the left visual field: HrHs (624.3 \pm 102.2$)$, HrLs (639.3 \pm 117.1$)$, LrHs (631.5 \pm 83.1), LrLs $(632.4 \pm 89.2)$; and in the right visual field: HrHs $(649.2 \pm 114.8)$, HrLs $(649.1 \pm 118.9)$, LrHs $(647.3 \pm 90.1)$, LrLs $(662.3 \pm 118.6)$.

Figure 4. Colour-coded overlap images of the lesions of neglect patients projected on axial sections of the MNI template brain with $z$-coordinates increasing from down $(z=-8)$ to up ( $z$ $=48$ ). The colour scales indicate the increasing number of overlapping lesions starting at $n=$ 1.

Figure 5. Topographic maps showing areas where theta-band functional connectivity was greater in controls than patients for A) the rDLPFC seed and B) the rSPC seed. Seed areas are shown in green. C) Spectrogram of the mean FC between the rSPC and rDLPC in the groups of controls and patients. Error bars represent standard deviations.

Figure 6. Correlations of the relevance index with theta-band functional connectivity between rSPC and rDLPFC in A) controls and distracters shown in the left visual field (LVF), B) controls and distracters shown in the right visual field (RVF), C) patients and distracters shown in the LVF and D) patients and distracters shown in the RVF. 
Figure 7. Voxel-wise linear correlation between theta-band functional connectivity and the behavioural relevance index for distracters appearing in the left (LVF) or right visual field (RVF). Regions (in red) correlated significantly with the relevance index and A) areas of controls' brain connected with the rDLPFC seed, B) areas of controls' brain connected with the rSPC seed, C) areas of patients' brain connected with the rDLPFC seed and D) areas of patients' brain connected with the rSPC seed. Seed areas are shown in green.

Figure 8. Correlations of the saliency index with theta-band functional connectivity between rSPC and rDLPFC in A) controls and distracters shown in the left visual field (LVF), B) controls and distracters shown in the right visual field (RVF), C) patients and distracters shown in the LVF and D) patients and distracters shown in the RVF. 
Table 1. Demographic and clinical characteristics of patients with varying degrees of spatial attention deficits.

\begin{tabular}{|c|c|c|c|c|c|c|c|c|c|c|c|c|}
\hline \multirow[b]{2}{*}{ Patients } & \multirow[b]{2}{*}{ Age } & \multicolumn{3}{|c|}{ Bells cancellation } & \multicolumn{2}{|c|}{ Reading } & \multirow{2}{*}{$\begin{array}{c}\text { Inverted T } \\
\text { cancellation : } \\
\text { Left omissions } \\
\text { (cut-off = 3) }\end{array}$} & \multirow{2}{*}{$\begin{array}{l}\text { Line bisection : } \\
\text { Ipsilesional } \\
\text { bias in \% } \\
\text { (cut-off }=4.8 \text { ) }\end{array}$} & \multirow[b]{2}{*}{$\begin{array}{l}\text { Lesion } \\
\text { volume } \\
\left(\mathrm{ccm}^{3}\right)\end{array}$} & \multicolumn{3}{|c|}{ Time since injury (days) } \\
\hline & & $\begin{array}{c}1^{\text {st }} \\
\text { cancellation } \\
\text { (lateralized } \\
\text { bias = right) }\end{array}$ & $\begin{array}{c}\text { Left } \\
\text { omissions } \\
\text { (cut-off = 3) }\end{array}$ & $\begin{array}{l}\text { Left-Right } \\
\text { omissions } \\
\text { difference } \\
\text { (cut-off = 2) }\end{array}$ & $\begin{array}{l}\text { Left omissions } \\
\text { (cut-off = } \\
28.9 \% \text { ) }\end{array}$ & $\begin{array}{l}\text { Left-Right } \\
\text { omissions } \\
\text { difference }\end{array}$ & & & & $\begin{array}{c}\text { Behavioral } \\
\text { testing }\end{array}$ & $\begin{array}{c}\text { EEG } \\
\text { recording }\end{array}$ & $\begin{array}{c}\text { Structural } \\
\text { imaging }\end{array}$ \\
\hline N1 & 69 & right & 3 & 2 & 6.25 & 2 & 3 & 6.2 & 89.9 & 36 & 56 & 37 \\
\hline N2 & 64 & right & 6 & 2 & 6.25 & 2 & 8 & 4.9 & 17 & 50 & 50 & 46 \\
\hline N3 & 64 & right & 15 & 9 & 81.25 & 10 & 20 & 23.1 & 159.2 & 30 & 29 & 2 \\
\hline N4 & 64 & right & 1 & 1 & 68.75 & 11 & 6 & 2.8 & 15.2 & 38 & 56 & 105 \\
\hline N5 & 41 & right & 7 & 6 & 18.75 & 4 & 8 & 4.2 & 107.2 & 25 & 74 & 88 \\
\hline N6 & 48 & right & 3 & 2 & 6.25 & 1 & 5 & 4.4 & 18.6 & 24 & 22 & 8 \\
\hline N7 & 54 & right & 8 & 6 & 100 & 18 & 27 & 10.7 & 30.13 & 52 & 84 & 51 \\
\hline N8 & 58 & right & 15 & 12 & 18.75 & 2 & 3 & 14.4 & 28.8 & 49 & 51 & 52 \\
\hline N9 & 81 & right & 15 & 11 & 50 & 3 & 6 & 6.5 & 150 & 51 & 76 & 42 \\
\hline N10 & 78 & right & 2 & -2 & 81.25 & 10 & 15 & 10.3 & 82.6 & 25 & 43 & 48 \\
\hline N11 & 63 & right & 3 & 2 & 6.25 & 1 & 2 & 7.8 & 15.6 & 38 & 37 & 24 \\
\hline $\begin{array}{l}\text { Patients } \\
\text { mean }\end{array}$ & $\begin{array}{c}62 \\
( \pm 11)\end{array}$ & - & $7.1( \pm 5.5)$ & $4.6( \pm 4.5)$ & $40.3( \pm 36.6)$ & $5.8( \pm 5.6)$ & $9.4( \pm 7.9)$ & $8.7( \pm 5.9)$ & $\begin{array}{c}64.4 \\
( \pm 55.4)\end{array}$ & $\begin{array}{c}38.0 \\
( \pm 11.1)\end{array}$ & $\begin{array}{c}52.5 \\
( \pm 19.6)\end{array}$ & $\begin{array}{c}45.7 \\
( \pm 30.4)\end{array}$ \\
\hline $\begin{array}{c}\text { Controls } \\
\text { mean }\end{array}$ & $\begin{array}{c}63 \\
( \pm 7)\end{array}$ & & & & & & & & & & & \\
\hline
\end{tabular}


A

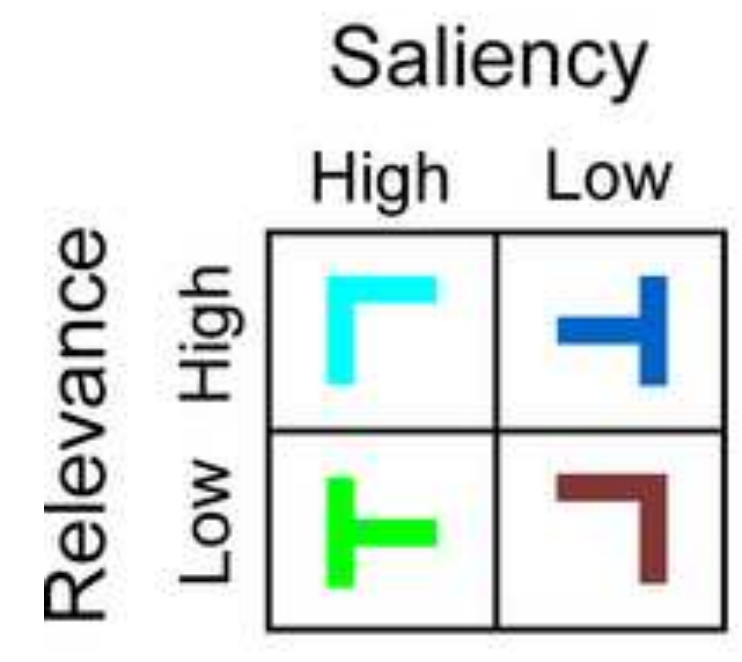

B

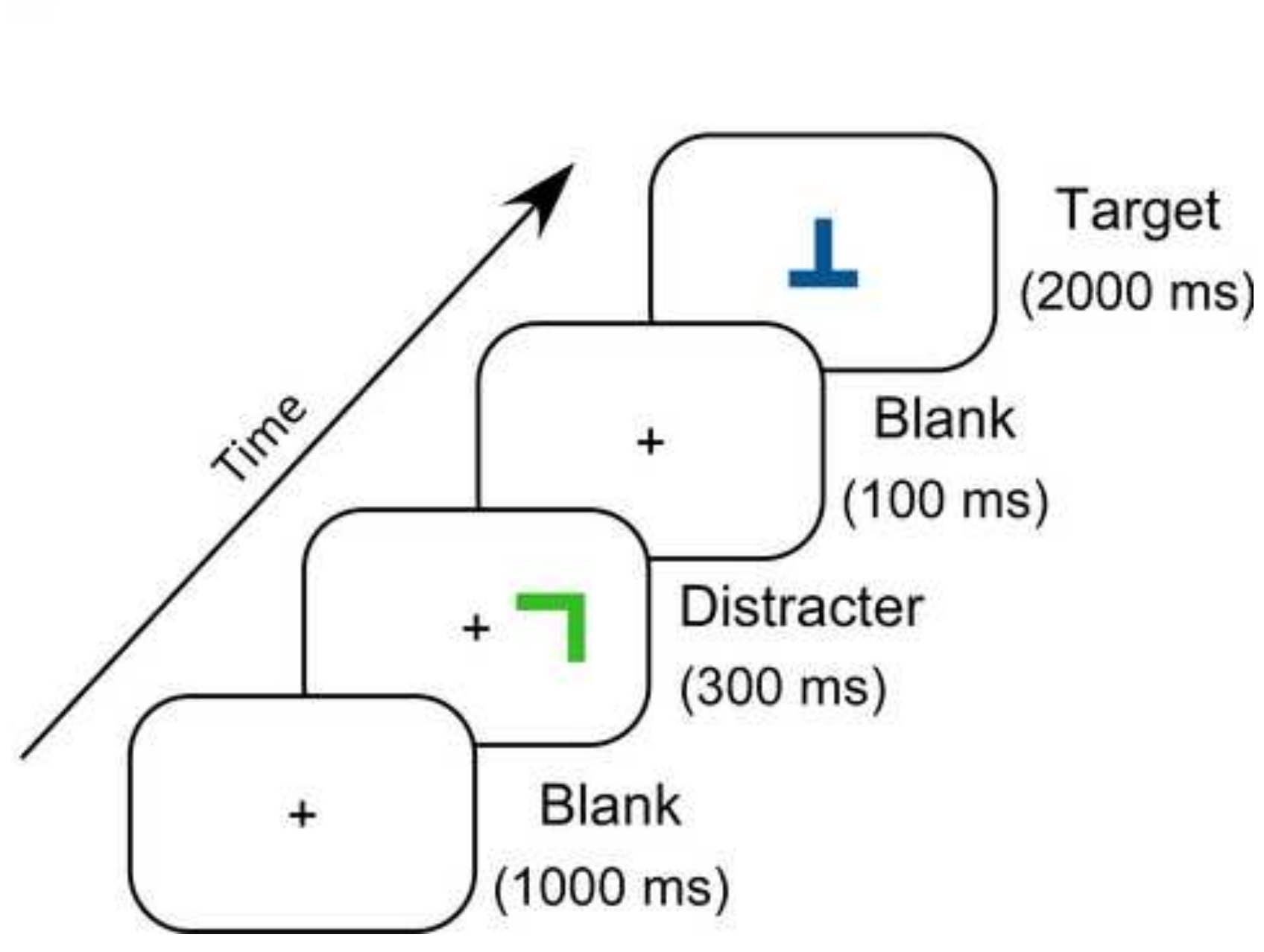



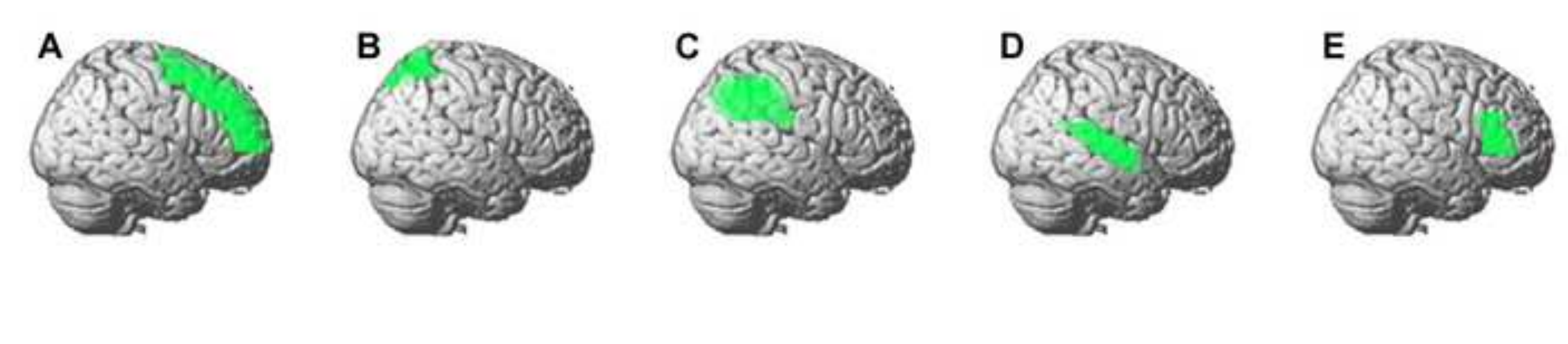
-O-Low saliency - - High saliency
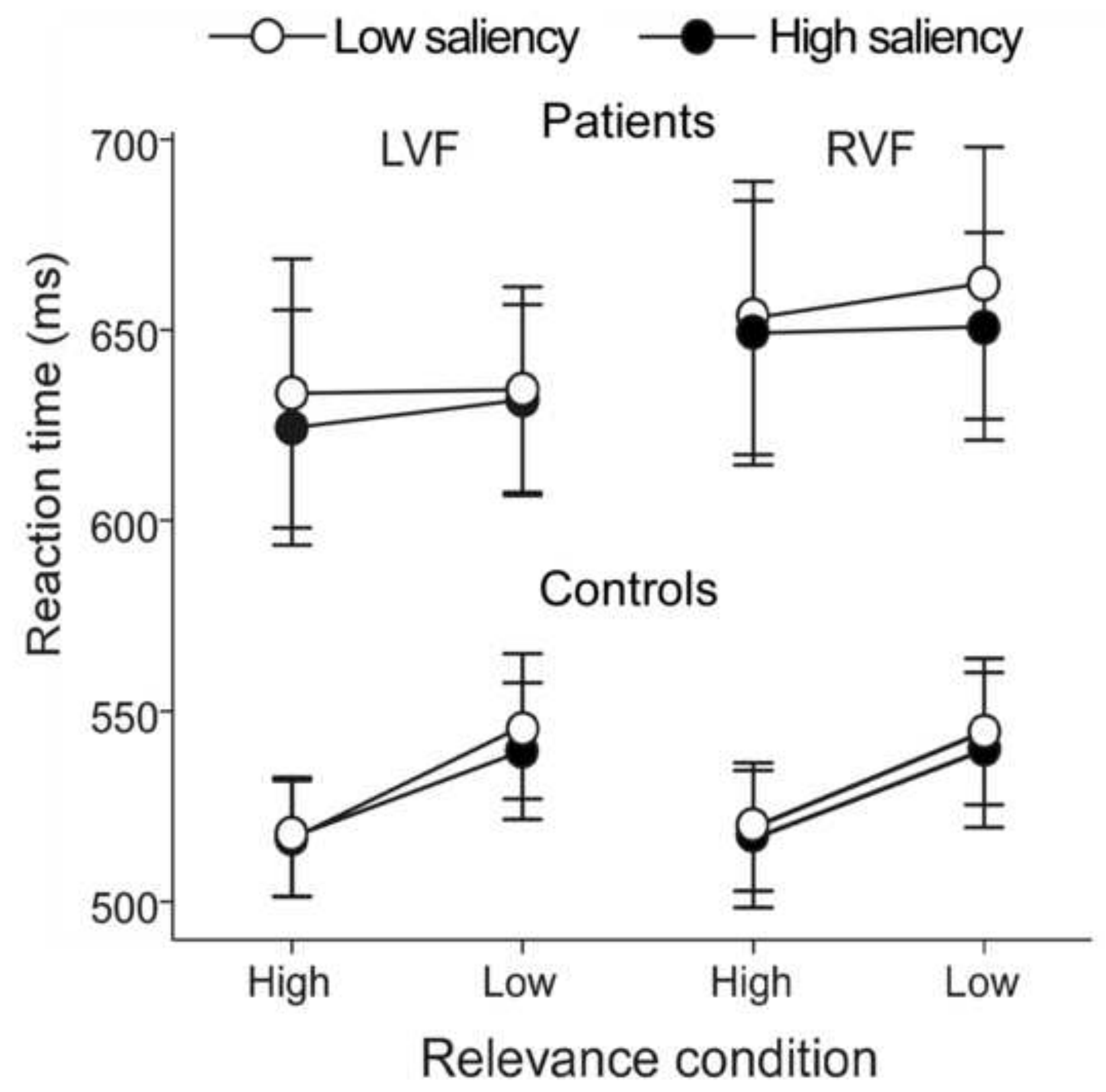

Relevance condition 
A

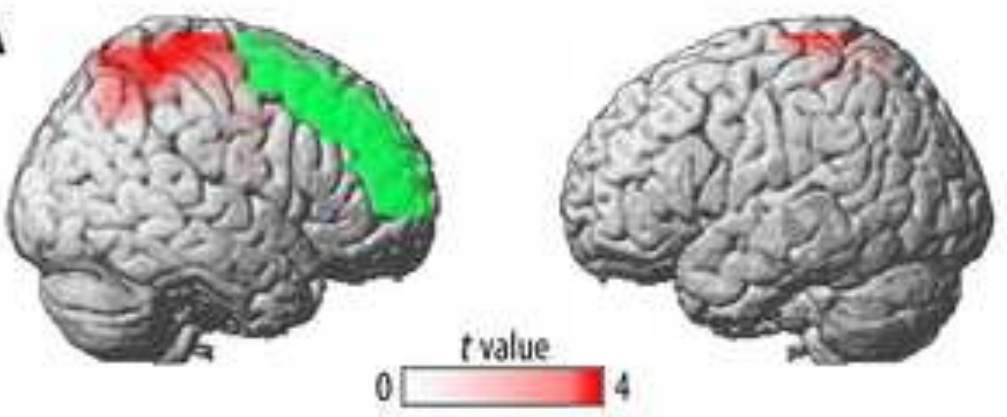

B

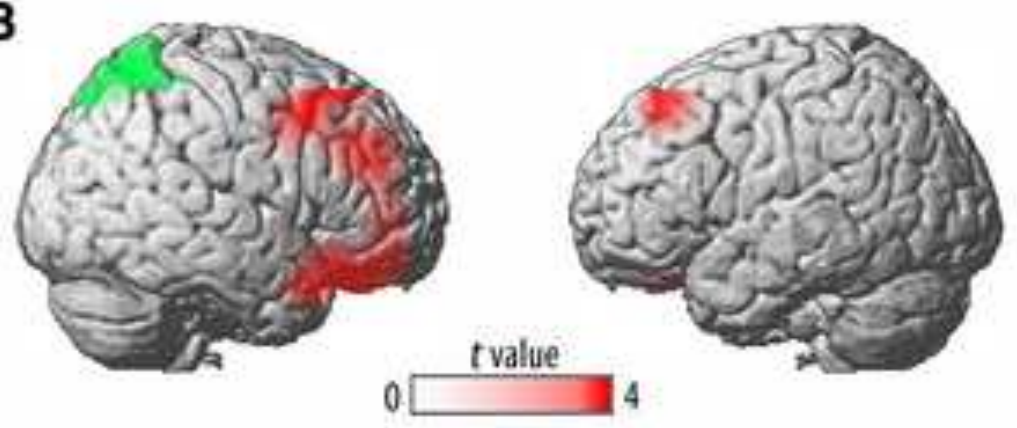

C

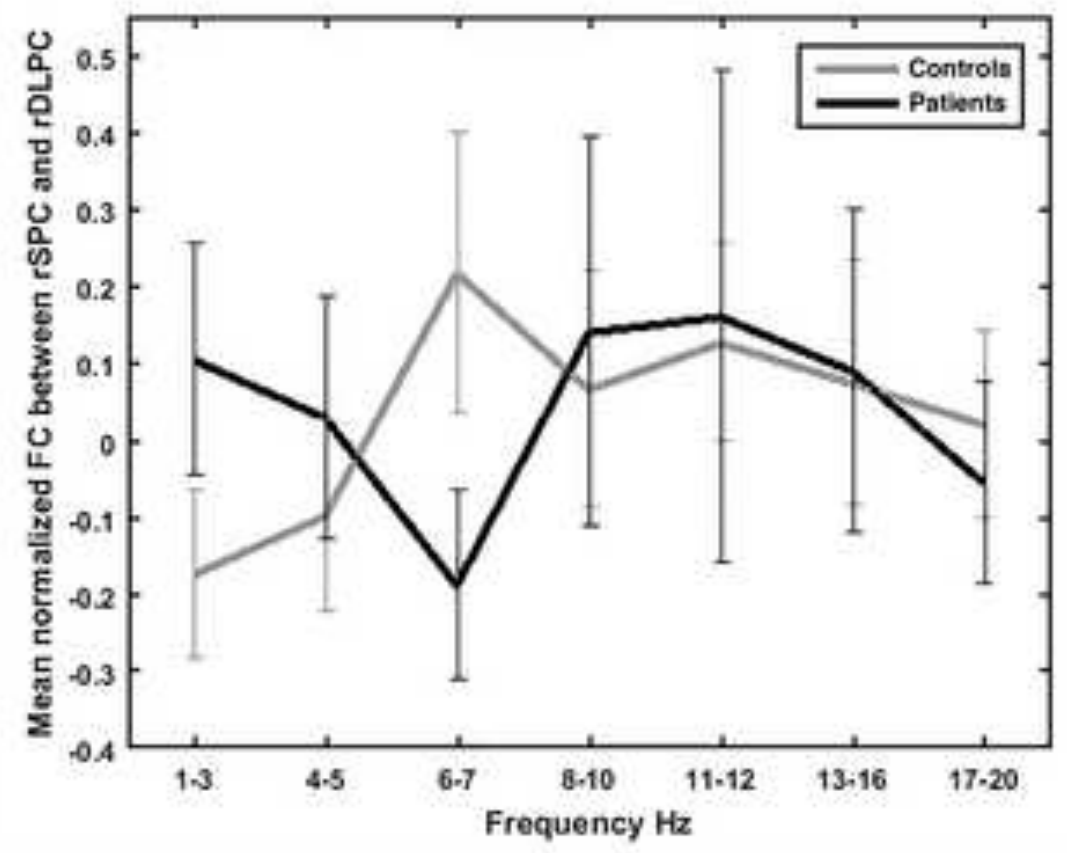




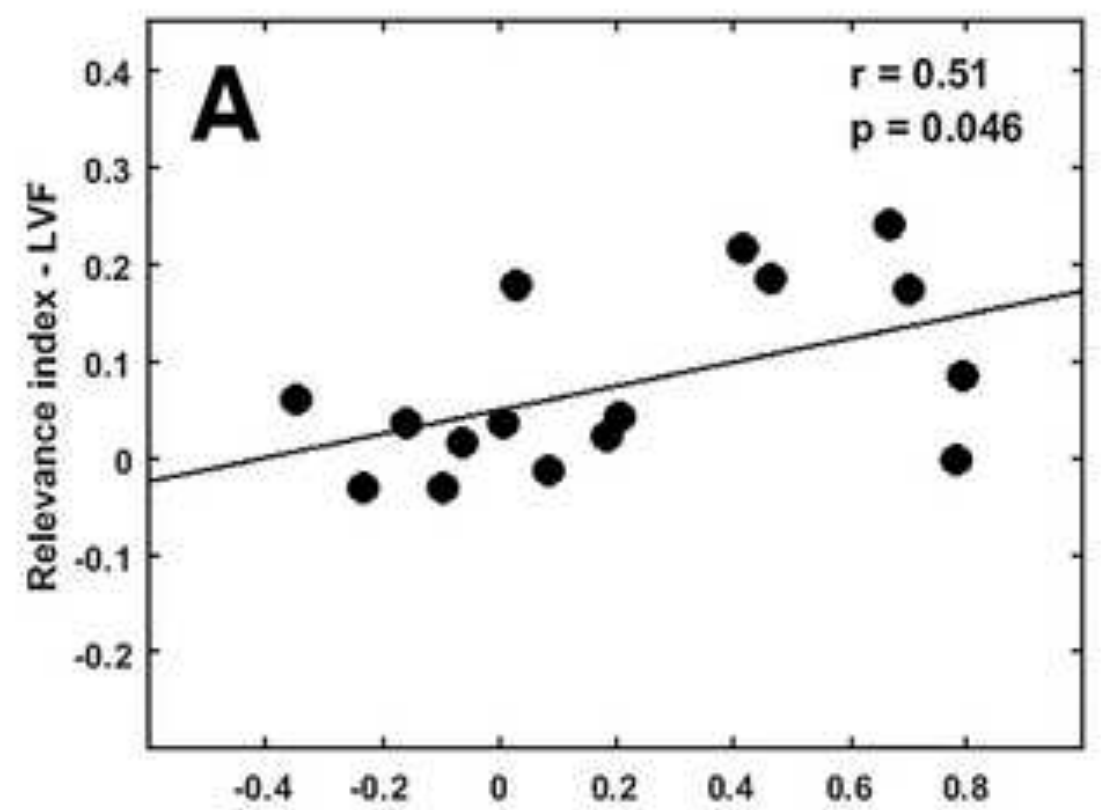

Mean normalized FC between rSPC and rDLPC

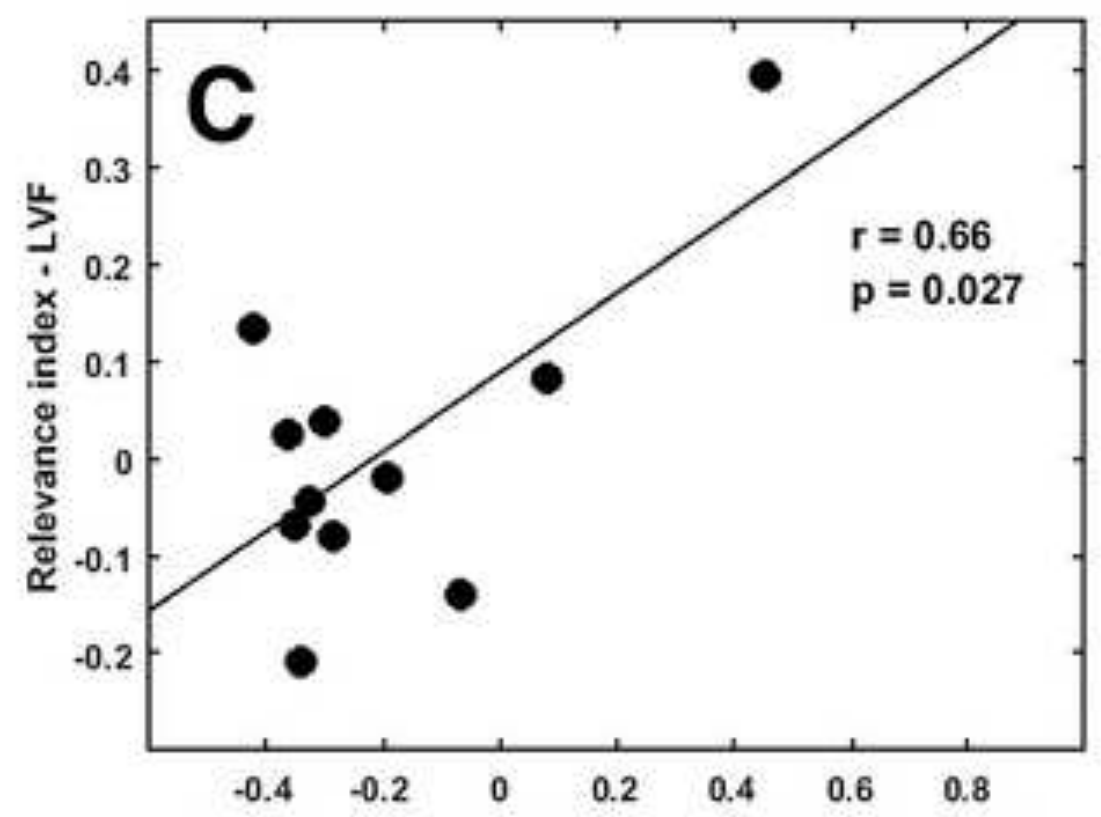

Mean normalized FC between rSPC and rDLPC

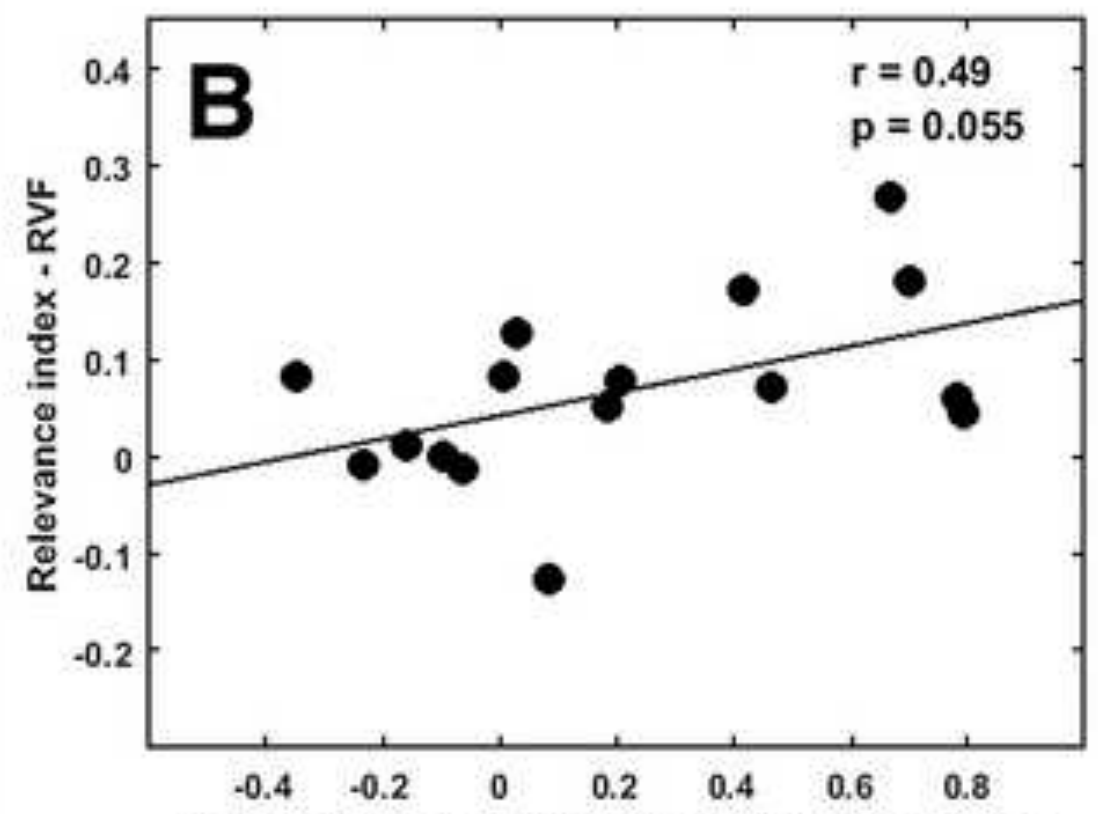

Mean normalized FC between rSPC and rDLPC

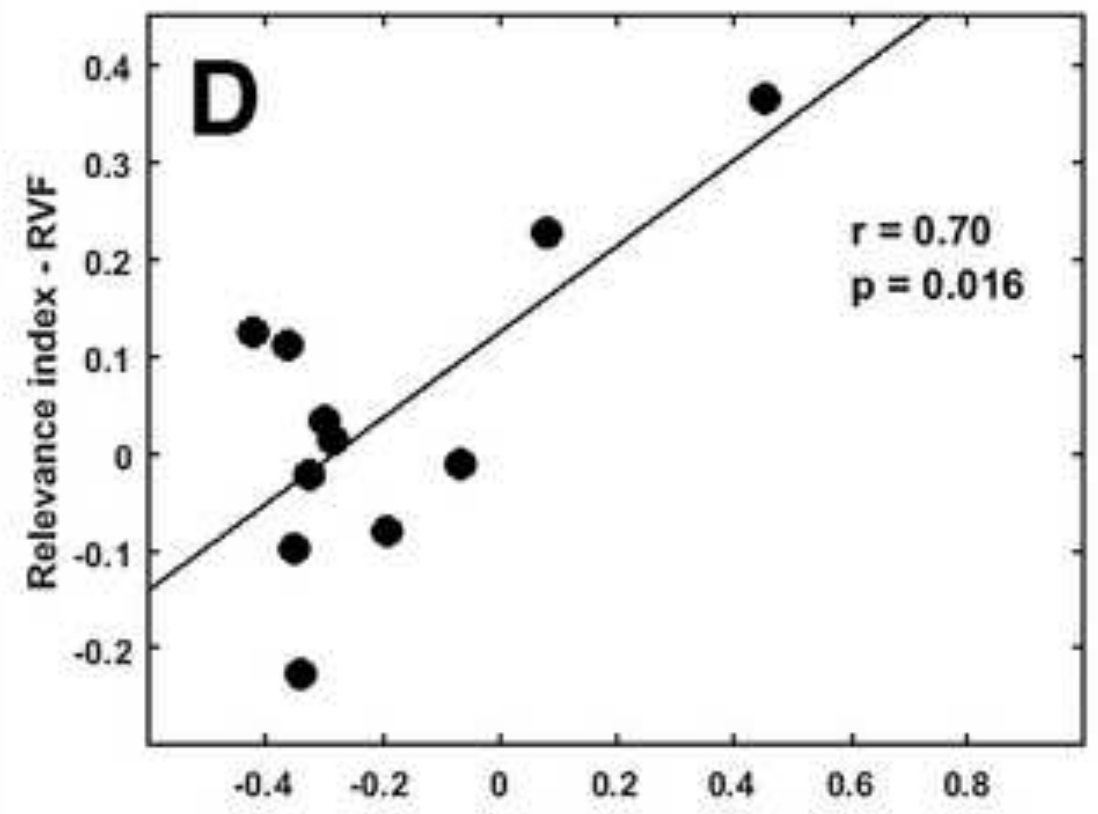

Mean normalized FC between rSPC and rDLPC 
LVF
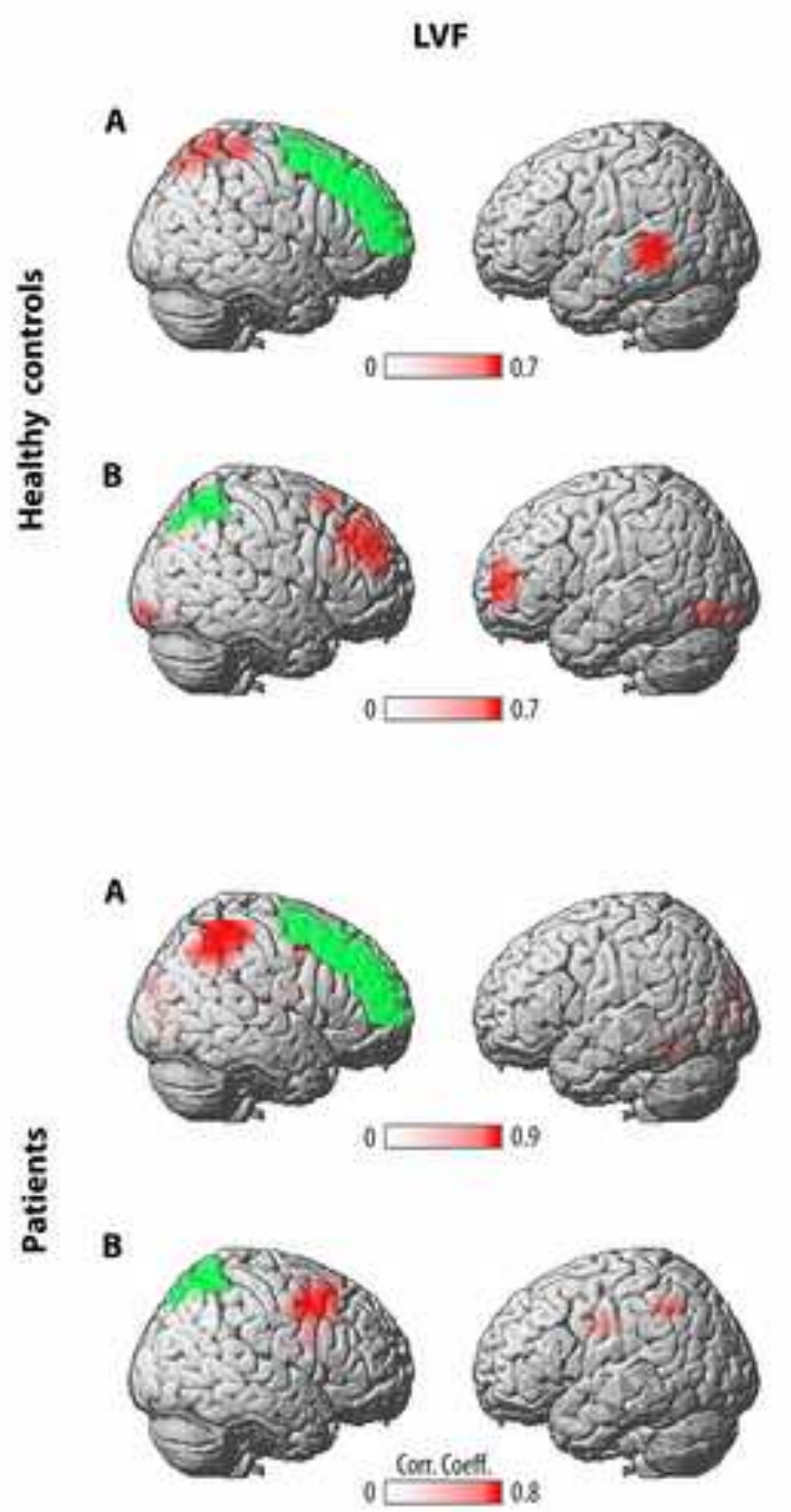

RVF
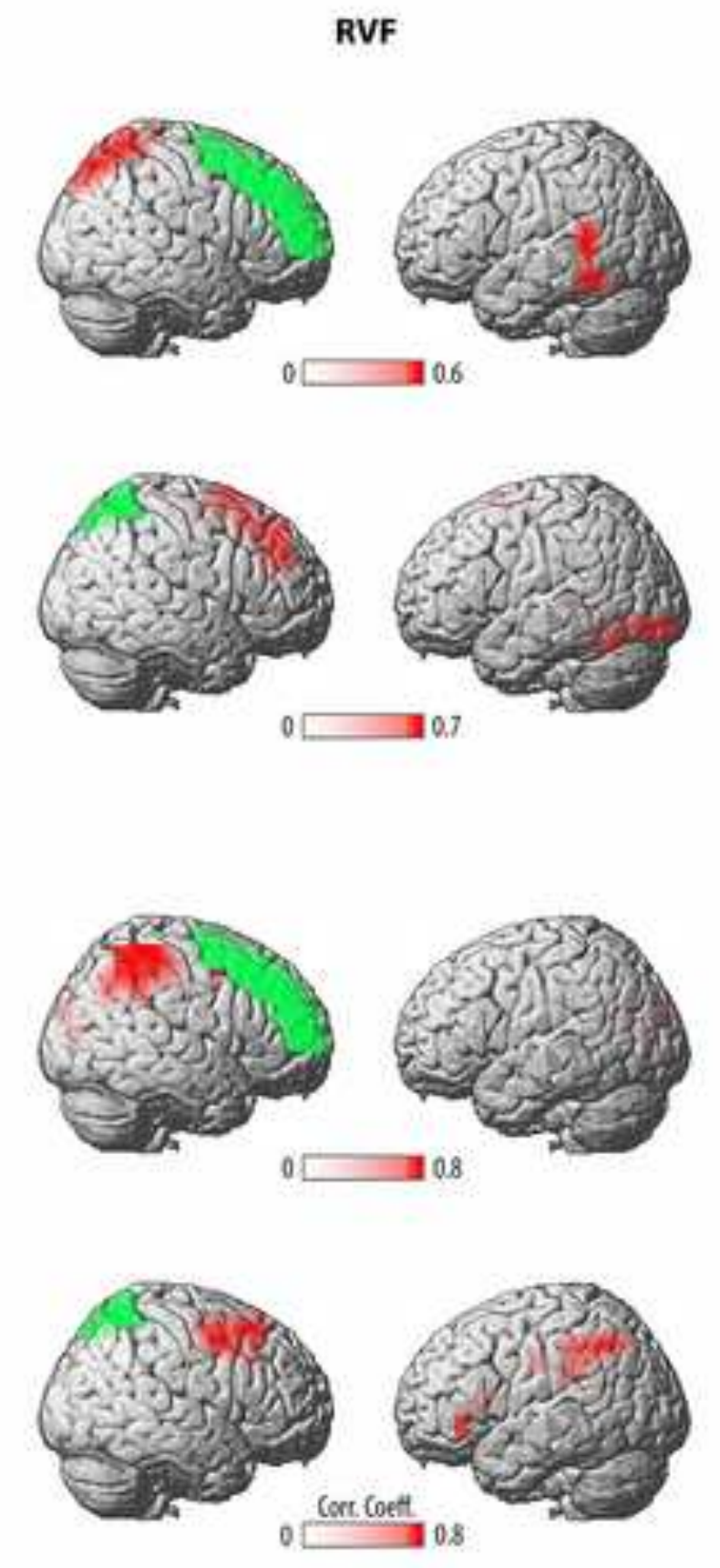

Figure7 


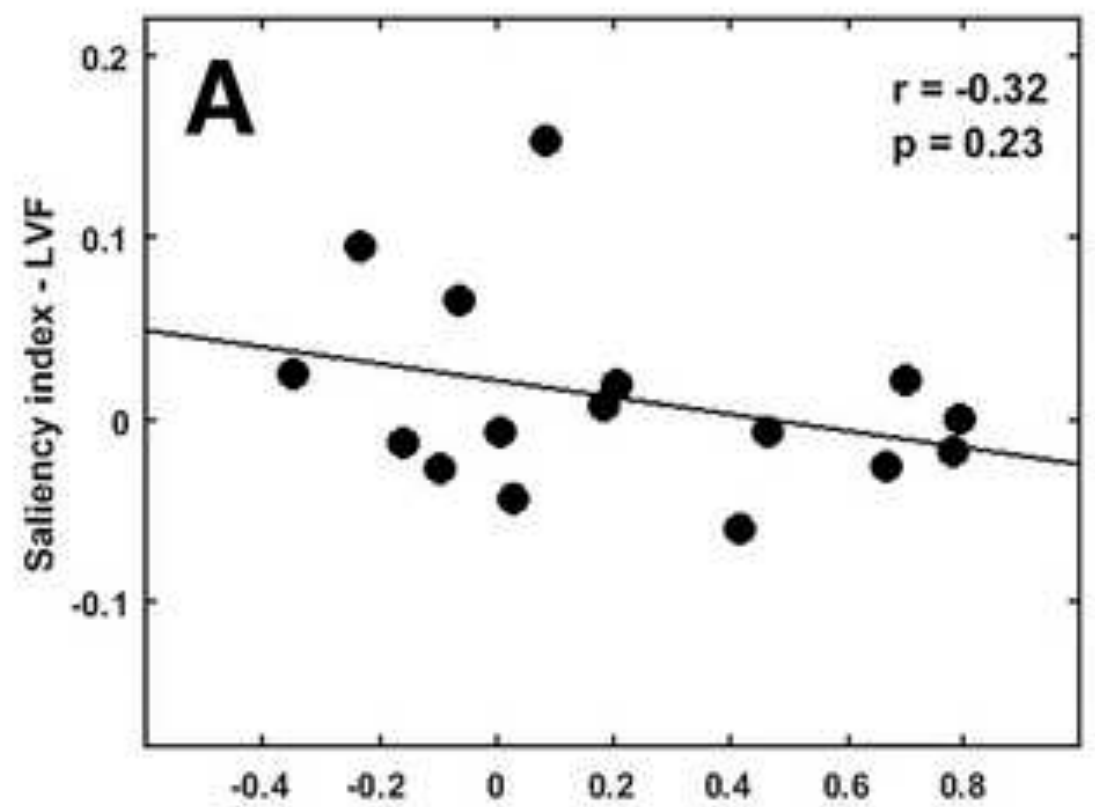

Mean normalized FC between rSPC and rDLPC

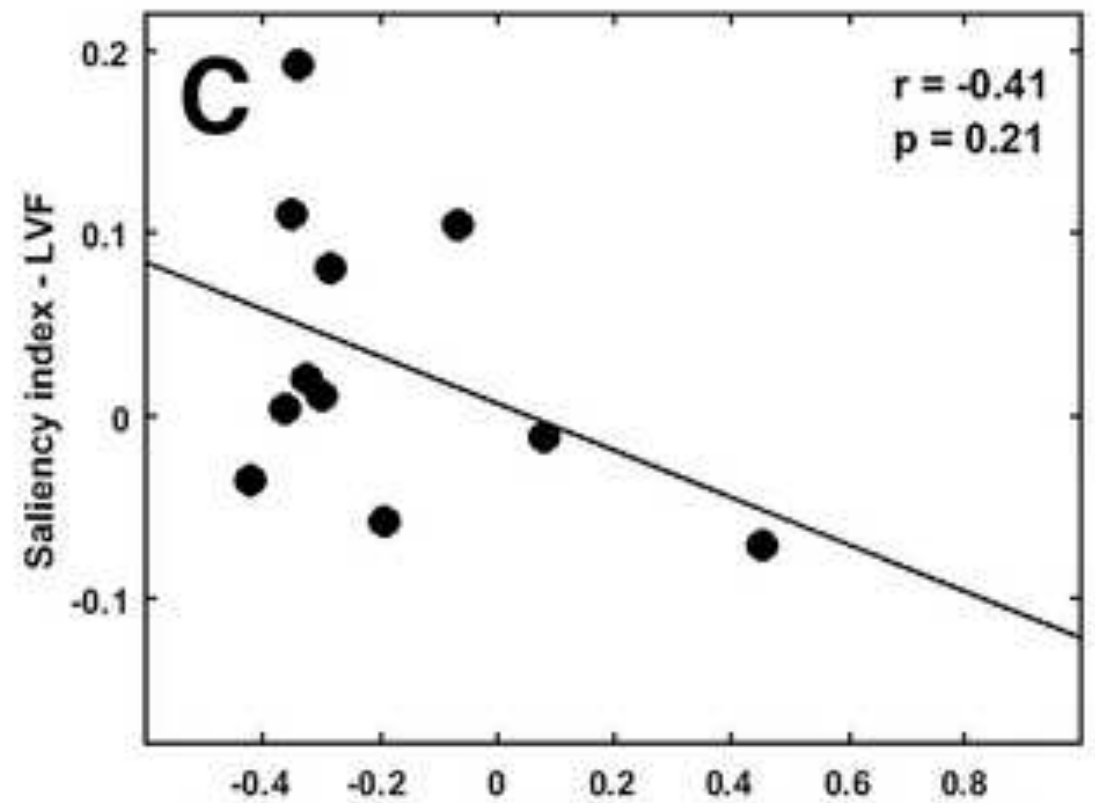

Mean normalized FC between rSPC and rDLPC

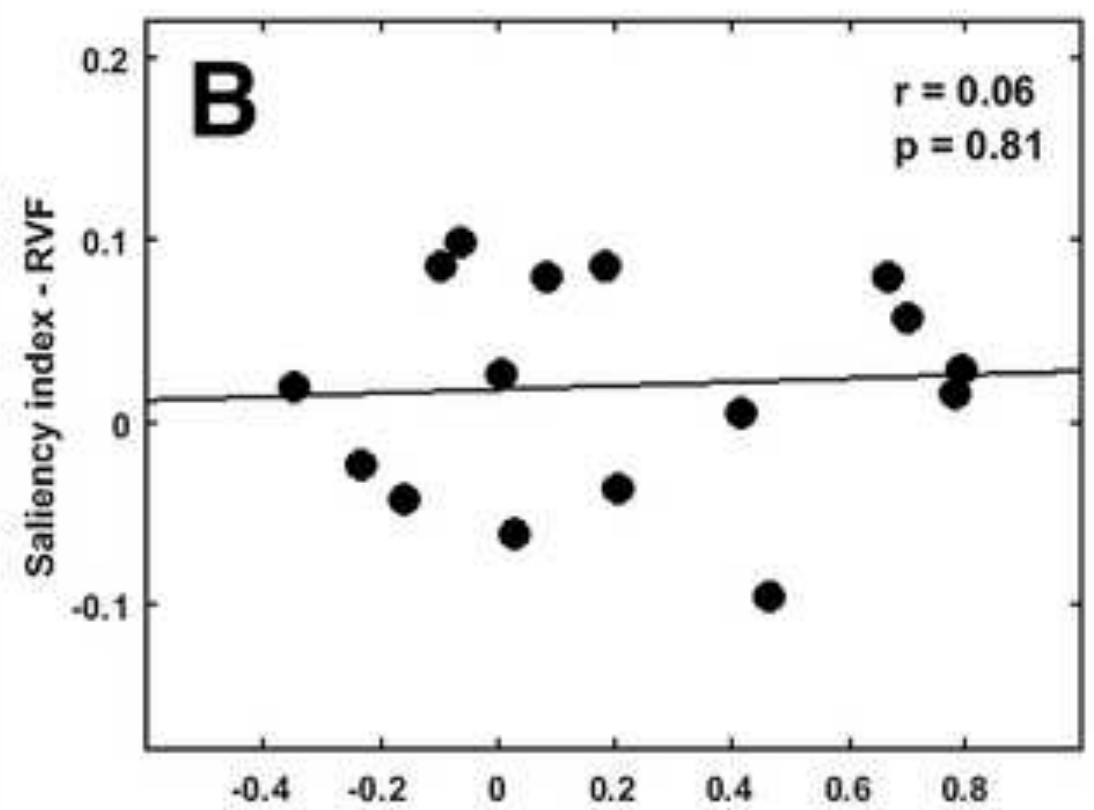

Mean normalized FC between rSPC and rDLPC

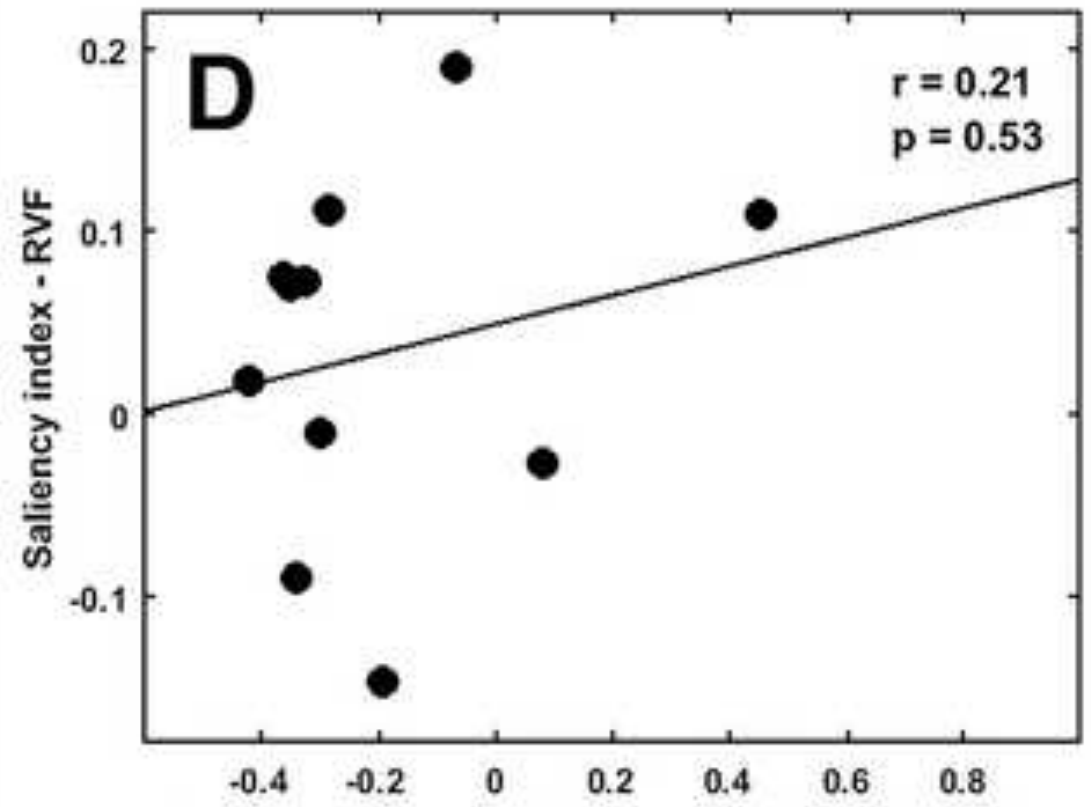

Mean normalized FC between rSPC and rDLPC 